\title{
Aplicación del Proceso Analítico Jerárquico en la comunicación digital de los organismos públicos durante la pandemia de la COVID-19
}

\author{
Application of the Analytical Hierarchical Process \\ in the digital communication of public agencies during the \\ COVID-19 pandemic
}

Eva Matarín Rodríguez-Peral. Universidad Rey Juan Carlos. España.

evamatarin@urjc.es

$[\mathrm{CV}] \odot \tau$

Tomás Gómez Franco ${ }^{1}$. Universidad Francisco de Vitoria. España.

tomas.gomez@ufv.es

$[\mathrm{CV}] \odot \mathrm{R}$

Almudena García Manso. Universidad Rey Juan Carlos. España.

almudena.manso@urjc.es

$[\mathrm{CV}] \odot \%$

Cómo citar este artículo / Referencia normalizada

Rodríguez-Peral, E. M., Gómez Franco, T. y García Manso, A. (2022). Aplicación del Proceso Analítico Jerárquico en la comunicación digital de los organismos públicos durante la pandemia de la COVID-19. Revista Latina de Comunicación Social, 80, 89-117. https://www.doi.org/10.4185/RLCS-2022-1532

\section{RESUMEN}

Introducción: esta investigación analiza la comunicación digital llevada a cabo por 21 organismos públicos del ámbito sanitario en la plataforma Twitter en el periodo de la pandemia de la COVID-19. Se centra en examinar los criterios de éxito de las estrategias de comunicación en esta red social digital y en conocer su peso ponderado en el modelo propuesto. Metodología: el registro de los datos se ha elaborado a través de la aplicación de acceso libre Twitonomy que analiza los últimos 3.200 Tweets de cada perfil. El análisis de la información se realiza aplicando el Analytic Hierarchy Process (AHP) o Proceso de Análisis Jerárquico como método para la toma de decisiones multicriterio. A través del software Expert Choice se estudian 17 criterios correspondientes a los clústeres actividad de la cuenta e impacto. Resultados: se determina la alternativa más adecuada en el ámbito de la comunicación de la salud pública en redes sociales y el peso de los criterios que benefician esta comunicación en la plataforma Twitter. Discusión y Conclusiones: este estudio confirma que es conveniente que los responsables de la toma de decisiones en materia de comunicación digital tengan presente que el clúster impacto tiene un mayor peso en la red social Twitter que la actividad de la cuenta.

\footnotetext{
${ }^{1}$ Autor responsable de la correspondencia
} 
PALABRAS CLAVE: salud pública; comunicación digital; Twitter; Proceso de Análisis Jerárquico; decisión multicriterio; COVID-19; redes sociales

\begin{abstract}
Introduction: this research analyses the digital communication carried out by 21 public health institutions on the Twitter platform during the period of the COVID-19 pandemic. It focuses on examining the criteria for the success of communication strategies on this digital social network and their weighted weight in the proposed model. Methodology: the data was recorded using the openaccess application Twitonomy, which analyses the last 3,200 Tweets from each profile. The analysis of the information is carried out by applying the Analytic Hierarchy Process (AHP) as a method for multi-criteria decision-making. Using Expert Choice software, 17 criteria corresponding to the account activity and impact clusters are studied. Results: The most appropriate alternative in the field of public health communication in social networks and the weight of the criteria that benefit this communication on the Twitter platform are determined. Discussion and Conclusions: this study confirms that it is advisable for decision-makers in digital communication to bear in mind that the impact cluster has a greater weight on the Twitter social network than account activity.
\end{abstract}

KEYWORDS: public health; digital communication; Twitter; Analytic Hierarchy Process (AHP); multi-criteria decision; COVID-19; social networks; social media

\title{
CONTENIDO
}

1. Introducción. 2. Objetivos. 3. Metodología. 4. Resultados. 5. Discusión/Conclusiones. 6. Bibliografía 7. Currículum Vitae

\section{Introducción}

En el ámbito de la salud el papel del paciente ha ido evolucionado hacia un rol activo que participa en la toma de decisiones (Pisano et al., 2021, p. 3) y en la búsqueda de información sanitaria. En España un $60 \%$ de la población utiliza Internet para buscar información en materia de salud (ONTSI, 2020). En los últimos 10 años se ha duplicado su uso, pasando de un $31 \%$ en el año 2009 a un $60 \%$ en el 2019 (Eurostat, 2020). Este rol de paciente activo y empoderado está estrechamente relacionado con la necesidad de obtener información veraz.

La pandemia producida por el SARS-CoV-2 ha derivado en situaciones de confinamiento y de distanciamiento físico que "han supuesto una modificación en las relaciones sociales" (FerrerSerrano et al., 2020, p. 3), dando lugar a nuevos hábitos de interacción a través de Internet y también a una mayor búsqueda de información en medios digitales y redes sociales (Cabrera y Clavijo, 2020, p. 130). Sin embargo, Internet tiene una faceta dual. Por un lado, es una gran fuente de información, pero por otro, en ocasiones facilita que se produzca infoxicación, dando lugar a conclusiones infundadas o erróneas (Navas-Martín et al., 2012, p. 155). El aumento en su uso como medio de información no siempre va acompañado de un aumento en la alfabetización digital que capacite al usuario en un uso eficiente de Internet y le permita discernir entre bulos e información verificada (Cabrera y Clavijo, 2020, p. 130). Ante esta situación, las organizaciones sanitarias se convierten en una fuente de referencia de información online, de manera cercana y sencilla, orientada a un público no especializado (Park et al., 2015, p. 1).

Durante la pandemia de la COVID-19 la plataforma Twitter se ha convertido en un canal relevante para organizaciones en diferentes contextos como el gubernamental, el sanitario o el educativo, entre 
otros. En el ámbito educativo la pandemia ha repercutido en el uso que han realizado de esta red social los centros escolares y las universidades, debido principalmente a su carácter colaborativo y a la posibilidad que esta plataforma brinda a las entidades de mostrar un papel activo (Ferrer-Serrano et al., 2020, p. 9). En este sentido, las investigaciones señalan que durante el periodo pandémico las universidades españolas han presentado un cambio en su comunicación digital, aumentando los mensajes que proyectan "un perfil cooperativo y pro-social" del centro (Ferrer-Serrano et al., 2020, p. 10). En el contexto sanitario y de salud pública Twitter también se ha convertido en una red muy relevante para la población debido a que ha facilitado la obtención de información de manera inmediata (Cabrera y Clavijo, 2020, p. 130)

Las entidades que utilizan en sus procesos de comunicación externa redes sociales digitales tienen que asumir decisiones estratégicas para llegar a la audiencia. Así, en la estrategia de comunicación de la red social Twitter se debe tener en cuenta qué objetivos comunicacionales tienen prioridad y mayor relevancia. Esto pudiera parecer a priori sencillo, sin embargo, cuando en la toma de decisiones entran en valor aspectos cualitativos y cuantitativos, surge la dificultad de tener que otorgar mayor relevancia a unos criterios sobre otros, dando lugar a un problema de decisión multicriterio (Guijarro et al., 2018, p.1415). Los responsables de comunicación de los organismos que llevan a cabo sus estrategias de comunicación digital en Twitter suelen tener que enfrentarse a este tipo de disyuntiva.

En los procesos de comunicación en los que se utilizan estos canales es conveniente llevar a cabo un análisis de las alternativas, evaluando la toma de decisión de criterios múltiples (MCDM) (Osorio, 2008, p. 247; Agwa-Ejon et al., 2017). El propósito de esta investigación es facilitar un modelo de decisión aplicable a la comunicación en Twitter de organismos públicos vinculados al ámbito de la salud, a través del Analytic Hierarchy Process (AHP) o Proceso de Análisis Jerárquico.

\subsection{La plataforma Twitter como canal de difusión de información vinculada a la salud}

La legislación española recoge el derecho de los ciudadanos a la información sanitaria. El artículo 29 de la Ley 12/2001 de 21 de diciembre, de Ordenación Sanitaria de la Comunidad de Madrid señala el derecho que tiene la ciudadanía a una "información sanitaria clara, veraz, relevante, fiable, equilibrada, actualizada, de calidad y basada en la evidencia científica, que posibilite el ejercicio autónomo y responsable de la facultad de elección y la participación activa" (Ley 12/2001). El uso masivo de las redes sociales ha tenido impacto en la comunicación en el ámbito sanitario, convirtiéndose en un canal de difusión de mensajes sobre salud que ha permitido ampliar el alcance y el acceso a esta información (Medina, 2018, p. 215).

El uso de estas redes sociales en el ámbito de la salud y más concretamente en contextos de crisis sanitarias como la producida durante la pandemia de la COVID-19, ha sido objeto de estudio de diversas investigaciones (Manfredi-Sánchez et al., 2021, p. 84; Pulido-Polo et al., 2021, p. 5; Zolbanin et al., 2021, p. 2; Bartlett et al., 2015, p. 379). El foco de interés se ha centrado en determinar el potencial que presentan las redes sociales como Twitter en la vigilancia de la salud pública, dado la cantidad de datos que facilita en tiempo real (Jordan et al., 2018, p. 2; Sinnenberg et al., 2017, p. e3); en aumentar el conocimiento sobre cómo se produce la comunicación de salud pública entre las organizaciones y las audiencias; los mensajes de los líderes políticos durante estos periodos; el estudio de las estrategias de comunicación que posibilitan que los mensajes obtengan un mayor alcance o, como en el caso de la investigación llevada a cabo por Zolbanin et al. (2021), en establecer si los mensajes sobre enfermedades publicados en redes sociales por los usuarios pueden se utilizados para determinar en un corto intervalo de tiempo la lista de los síntomas de una enfermedad (p. 1). 
Dentro de las redes sociales "Twitter es, sin duda, la red social más influyente en el ámbito sanitario, y en la que autores, organismos científicos, profesionales y pacientes centran la mayor parte de su atención" (Barajas-Galindo y Rodríguez Carnero, 2020, p. 295). Obteniendo una elevada popularidad entre los líderes políticos y la ciudadanía (Caldevilla-Domínguez et al., 2019, p.1288). Prueba de ello es el uso que los presidentes de los distintos países han hecho de la comunicación digital a través de esta plataforma para poner al día a la población de las novedades frente a la COVID-19 (ManfrediSánchez et al., 2021, p. 86).

En entornos de elevada incertidumbre, como la derivada de este contexto pandémico, se evidencia la relevancia que posee la comunicación y que la población esté debidamente informada ante una situación de crisis sanitaria. El uso de este tipo de plataformas sociales con el objetivo de llevar a cabo una comunicación sanitaria puede facilitar la participación social, la interacción con la audiencia y el apoyo informativo, empoderando a los usuarios en su toma de decisión en materia de salud (Centers for Disease Control and Prevention, 2011, p. 1; Myrick et al., 2016, p. 5).

Los datos de infodemiología, entendida esta como la profusión de información en Internet, centrados en el ámbito de la comunicación sanitaria en Twitter, indican que esta red permite a los profesionales conocer en tiempo real la opinión, percepción o sensación de los usuarios (Pulido-Polo et al., 2021, p. 10; OMS, 2020; Eysenbach, 2020, p. 2; Schillinger et al., 2020, p.1393). De este modo, es factible la obtención de métricas vinculadas a la actitud de la audiencia ante un servicio público o privado (Eysenbach, 2011, p. S157), con la repercusión que el tratamiento de esta información puede conllevar en la mejora o impulso de un servicio en un sector determinado. En materia de salud pública Twitter también se ha convertido en un medio útil para la obtención de información sobre los primeros síntomas de una enfermedad ante el posible brote de una pandemia (Guo et al., 2021, p. 7). Asimismo, son relevantes en la gestión de una pandemia los análisis que hacen referencia al sentimiento de los mensajes que los usuarios expresan en sus redes sociales. En este sentido, los estudios han señalado que durante el inicio de la campaña de vacunación contra la COVID-19, en Twitter han prevalecido los mensajes negativos (Hou et al., 2021, párr. 12). Este tipo de estudios ofrecen información sobre la necesidad de impulsar campañas que aumenten la confianza de la población en el proceso de vacunación.

El análisis de las publicaciones en redes sociales digitales en materia de sanidad facilita la comprensión de la comunicación existente entre los organismos oficiales y el resto de los participantes. Este conocimiento puede ser útil en momentos de crisis sanitarias "para orientar las futuras estrategias de comunicación de crisis de salud emergentes globales" (Wang et al., 2021, p. 11). Las investigaciones sobre la gestión de pandemias evidencian la relevancia que tiene poder monitorizar la información del entorno para obtener información que facilite el impulso de medidas de prevención ante una posible crisis sanitaria, pero, además, es preciso que los responsables en comunicación aporten información a la audiencia para reducir la ansiedad y el estrés del público (Xifra, 2020, p. 3). La mayoría de los mensajes orientados a impulsar un conocimiento de la salud tienen su origen en organizaciones sin ánimo de lucro $(\mathrm{ONG})(49,2 \%)$ (Park et al., 2013, p. 415). A pesar de que en el ámbito sanitario se está generalizando el uso de redes sociales, su uso aún es poco efectivo, dado que suele observarse ausencia de bidireccionalidad en la comunicación; falta de interactividad y ausencia de profesionalidad en la gestión de las cuentas (Sendrá-Toset y FarréComá, 2016, p. 293). Sin embargo, la información publicada en Twitter sobre la salud por parte de organismos gubernamentales es organizada de una manera que facilita en mayor medida la comprensión por parte del público (45,5\%) (Park et al., 2013, pp. 415-418). 
Los estudios sobre imagen corporativa de los hospitales españoles indican que deben profesionalizar su comunicación en las redes sociales digitales, potenciando su presencia en las mismas y planteándose unos objetivos comunicacionales (Medina, 2018, p. 222). Estos hospitales señalan que entre las principales dificultades para llevar a cabo la comunicación por redes sociales se encuentra la falta de formación en la gestión de la comunicación; actualización de los contenidos; implicación de los profesionales. Asimismo, se observa dificultad para llegar a los potenciales usuarios y ausencia de retroalimentación ciudadana (Rando y de las Heras, 2016, p. 568). El análisis de la comunicación corporativa de los hospitales andaluces señala que cerca del 60\% de los que utilizan las redes sociales para realizar su comunicación sanitaria buscan promover una comunicación eficaz con la sociedad (Rando y de las Heras, 2016, p. 566). La mayoría, además, lo hace para favorecer la proyección social y difundir información. En menor medida, pero también un aspecto relevante es el objetivo de impulsar una imagen de cercanía de los profesionales sanitarios (Rando y de las Heras, 2016, p. 565). Todo ello evidencia la necesidad de abordar la comunicación sanitaria desde una orientación estratégica, planificada y profesional, con mensajes adaptados al medio y a la audiencia.

Las instituciones sanitarias tienen el desafío de conocer el interés que generan en la audiencia las publicaciones que realizan en redes sociales digitales. La relevancia de la interacción del público con estas publicaciones radica en conocer los aspectos clave que facilitan que se genere dicho interés, impulsen la colaboración entre los usuarios de las redes y el intercambio rápido y efectivo de información (Sanz-Lorente et al., 2018, p. 5).

\subsection{El Proceso de Análisis Jerárquico en la toma decisiones}

Durante la década de los 70 Thomas L. Saaty desarrolló el Analytic Hierarchy Process (AHP) o Proceso de Análisis Jerárquico, un método para facilitar la toma de decisión en cuestiones complejas en las que intervienen múltiples criterios (Paredes, 2010, p. 96), como pueden ser aspectos cualitativos difíciles de valorar para poder determinar una decisión, como cuantitativos.

En la toma de decisión este proceso requiere de la evaluación de cada uno de los criterios que intervienen, con el objetivo de proporcionar un modelo jerárquico de prioridades para cada opción (Hurtado y Bruno, 2005; Lamirán-Palomares et al., 2020, p. 7). En cierto modo, consiste en incluir en la toma de decisión la cuantificación o el valor numérico de elementos cualitativos.

El AHP segmenta el problema para poder observar con mayor claridad las posibles soluciones. Su respuesta no es discrecional, sino que proporciona alternativas basándose en los criterios establecidos y jerarquizados de antemano.

Es la manera de aportar un soporte matemático a la toma de decisión utilizando criterios cuantitativos y cualitativos en una misma escala (Hurtado y Bruno, 2005). La dificultad previa para llevar a cabo la toma de decisión se debe, además, a que en múltiples ocasiones los criterios son contrarios y se genera conflicto (Yepes, 2018). Por ello, se desglosa el problema jerarquizándolo. La figura 1 muestra la disposición de los criterios en un Proceso Analítico Jerárquico. 


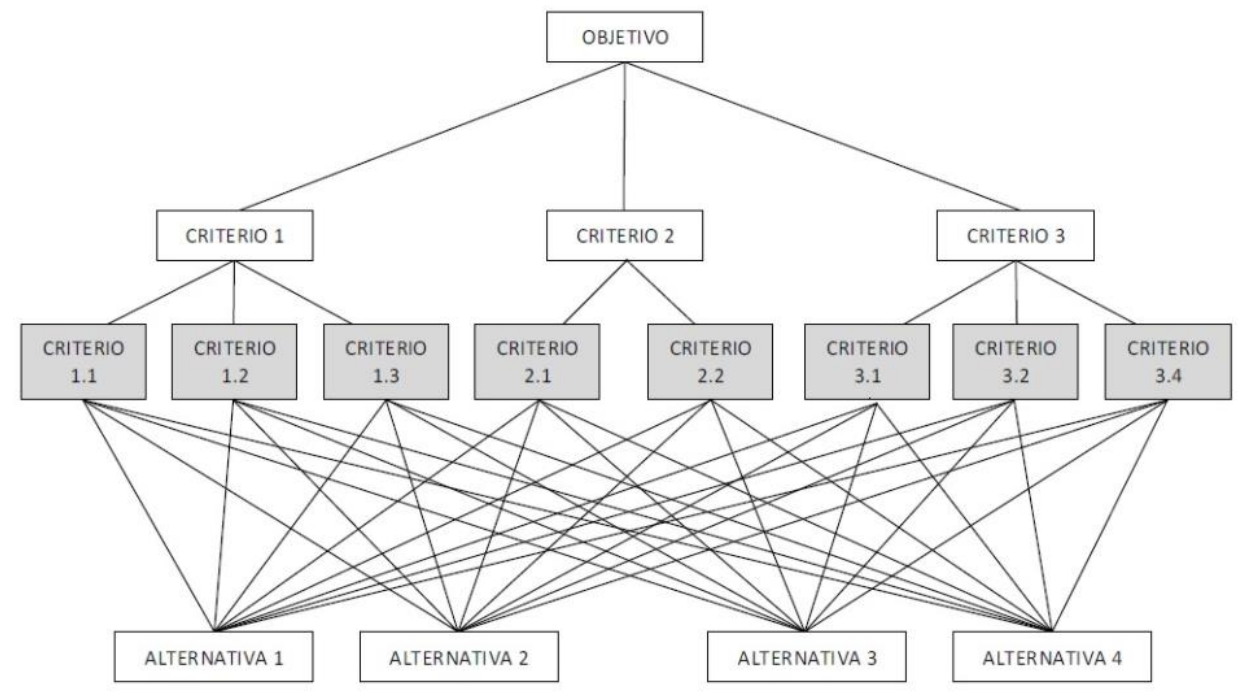

Figura 1: Ejemplo de Estructura Jerárquica AHP

Fuente: Yepes (2018). https://cutt.ly/9bJyhr7

Se establece una jerarquía en la que el objetivo que se pretende alcanzar se encuentra situado en la cúspide, seguido por debajo de los criterios, subcriterios y alternativas con la idea de que se pueda llevar a cabo de manera sencilla la comparación entre criterios del mismo nivel (Yepes, 2018). En su elaboración se suele utilizar la escala de preferencias que se muestra en la Tabla 1, conocida como la escala fundamental de comparación por pares (Saaty, 1980).

Tabla 1. Escala fundamental de comparación por pares

\begin{tabular}{lll}
\hline Valor & Definición & Comentarios \\
\hline 1 & Igual importancia & El criterio A es igual de importante que el criterio B \\
\hline 3 & Importancia moderada & $\begin{array}{l}\text { La experiencia y el juicio favorecen ligeramente al criterio A } \\
\text { sobre el B }\end{array}$ \\
\hline 5 & Importancia grande & $\begin{array}{l}\text { La experiencia y el juicio favorecen fuertemente al criterio A } \\
\text { sobre el B }\end{array}$ \\
\hline 7 & Importancia muy grande & El criterio A es mucho más importante que el criterio B \\
\hline 9 & Importancia extrema & $\begin{array}{l}\text { La mayor importancia del criterio A sobre el B está fuera de } \\
\text { toda duda }\end{array}$ \\
\hline $2,4,6$ y 8 & Valores intermedios entre los anteriores, cuando es necesario matizar \\
\hline
\end{tabular}

\section{Fuente: Saaty (1980).}

Como se observa en los estudios sobre toma de decisiones con técnicas multicriterio en la elaboración de modelos de análisis de factores de éxito, esta escala se continúa implementando en la actualidad (Guijarro et al., 2018, p. 1419; Velastegui et al., 2020, pp.161-162). Se utiliza para poder llevar a cabo la comparación pareada entre criterios, cuantificando los elementos cualitativos. Los pasos para llevar a cabo este tipo de procesos son, en primer lugar, establecer el peso de cada criterio y posteriormente comparar las alternativas existentes para cada criterio, ponderando los resultados (González, 2019).

El estudio de los marcos analíticos sobre los aspectos que aportan éxito y popularidad en la comunicación tecnológica puede abordarse a través del estudio de modelos multicriterio vinculados al análisis de la comunicación en Twitter de las organizaciones. En esta línea existen investigaciones 
orientadas a la comunicación externa de organismos públicos vinculados a perfiles de seguridad como la llevada a cabo por Guijarro et al. (2018).

Debido a que Twitter es una plataforma relativamente nueva, surgió en el año 2006, los estudios que versan sobre ella son actuales y existe un amplio espacio en el que avanzar indagando en el análisis de las estrategias de comunicación en esta red. El propósito de este estudio es aportar información que facilite la selección de los elementos más relevantes que deben ser tenidos en consideración en los procesos de comunicación en Twitter.

Este estudio hace frente a la cuestión de los elementos que aportan éxito y popularidad en la comunicación a través de una investigación, enmarcada en el análisis de la comunicación en materia de salud, que tiene como propósito medir las variables principales que utilizan los perfiles sanitarios para influir en la sociedad.

\section{Objetivos}

El objetivo general de esta investigación es aportar una mayor comprensión sobre la influencia que tienen las redes sociales digitales en el ámbito de la salud. Proporcionando una herramienta que facilite a las instituciones la toma de decisiones de carácter cualitativo en estos procesos de comunicación en Twitter, aplicando el Analytic Hierarchy Process (AHP).

Este estudio presenta como objetivos específicos elaborar un modelo de análisis jerárquico siguiendo las líneas de investigación de autores/as que han reorientado este modelo al ámbito de las redes sociales (Guijarro et al., 2018; Lamirán-Palomares et al., 2020), así como evaluar su validez en el caso de perfiles institucionales del ámbito de la salud pública, determinando el peso de los criterios que tienen una mayor influencia en Twitter y estableciendo qué perfiles institucionales públicos, vinculados a temas de salud, presentan un mayor peso en los criterios propuestos por este modelo.

\section{Metodología}

Para conseguir estos objetivos se ha planteado analizar el uso de Twitter que realizan 21 organismos públicos del ámbito sanitario español, así como la valoración de los criterios de éxito de su estrategia de comunicación en la red, a través del modelo multicriterio, siguiendo la línea de investigación planteada por las autoras Guijarro et al., (2018).

Se ha seleccionado Twitter, por su relevante número de usuarios y porque es una plataforma en la que la información que prima es principalmente de índole informativa. En este sentido, se han descartado otras aplicaciones con más usuarios que Twitter, como es el caso de Facebook, debido a que, aunque esta última permite su uso por organizaciones, es una red social más enfocada a las relaciones personales.

Este estudio pretende analizar la comunicación en salud llevada a cabo por la administración pública. Por este motivo se ha considerado prioritario analizar esta red social, dado que la totalidad de los mensajes de esta red son de acceso público.

La unidad de análisis es el tweet y el ámbito de estudio son los tweets publicados por perfiles institucionales españoles del ámbito de la salud pública. En la selección de perfiles se han establecido dos niveles, uno determinado por el carácter nacional de los perfiles y otro que viene definido por el carácter regional. Se tiene en cuenta que el público al que se dirige cada uno de los perfiles y el alcance de la información es diferente en el eje nacional y regional. De este modo, queda 
recogido todo el abanico de información institucional transmitida en materia de salud pública. La comunicación de los perfiles regionales es una información cercana, centrada principalmente en el territorio, mientras que los perfiles nacionales están orientados al conjunto de la población. En este sentido, también se han tenido en cuenta aspectos que pueden influir en el alcance de la comunicación, como es el idioma utilizado, dado que algunas cuentas regionales han establecido su información en varios idiomas. De este modo, se han monitorizado los 21 perfiles de usuarios que se detallan en la tabla 2.

Tabla 2. Perfiles de los usuarios de Twitter analizados

\begin{tabular}{ll}
\hline Nivel de representación nacional en el ámbito de sanitario & \\
\hline $\begin{array}{l}\text { Ministerio de Sanidad, Consumo y Bienestar Social: Min. Sanidad } \\
\text { (MSCBS) }\end{array}$ & @ sanidadgob \\
\hline Instituto Nacional de Gestión Sanitaria (INGESA) & \\
\hline Nivel de representación regional en el ámbito de sanitario & @SanidadCLM \\
\hline Sanidad Castilla La Mancha & @Salud_JCYL \\
\hline Salud Castilla y León & @ GVAsanitat \\
\hline Consejería Sanidad de Valencia & @SanidadGobCan \\
\hline Consejería Sanidad de Canarias & @ JuntaEx_Sanidad \\
\hline Sanidad Junta de Extremadura & @ saludand \\
\hline Consejería de Salud y Familias de la Junta de Andalucía & @SaludMadrid \\
\hline Consejería de Sanidad de la Comunidad de Madrid & @ astursalud \\
\hline Portal de Salud del Principado de Asturias & @osakidetzaEJGV \\
\hline Servicio Vasco de Salud & @SaludPublicaAra \\
\hline Dir Gral de Salud Pública Aragón & @icscat \\
\hline Institut Català de la Salut. Departament de Salut. Generalitat de & \\
Catalunya & @ snsalud_na \\
\hline Espacio de Salud del Gobierno de Navarra & @ibsalut \\
\hline Servei de Salut (IB-Salut) Govern de les Illes Balears & @Murciasalud \\
\hline Consejería de Salud de Murcia & @ riojasalud \\
\hline Servicio de Salud de la Rioja & @SanidadeXunta \\
\hline Perfil oficial da Consellería de Sanidade da Xunta de Galicia & @ saludcantabria \\
\hline Consejería de Sanidad del Gobierno de Cantabria & @ Ingesa_Ceuta \\
\hline Instituto Nacional de Gestión Sanitaria en Ceuta & @Ingesa_Melilla \\
\hline Instituto Nacional de Gestión Sanitaria en Melilla & \\
\hline
\end{tabular}

Fuente: elaboración propia a partir de información de Twitter

El universo está compuesto por todos los tweets que cada una de las cuentas de estos perfiles han difundido en Twitter desde que fueron creadas. La dificultad que conlleva el análisis de los mensajes en Twitter es, como señalan los investigadores Pérez et al., (2013) que "Twitter es un buen vocero, pero un nefasto archivero" (p. 11). La extracción de su información está limitada al número de tweets. El espacio temporal seleccionado es determinado por la disponibilidad de los datos a través de la herramienta gratuita Twitonomy que analiza los 3.200 tweets más recientes de cada uno de los usuarios. La extracción del contenido de los 21 perfiles estudiados se realizó durante la primera semana de diciembre del año 2020. Se ha considerado adecuado utilizar una muestra de datos en plena crisis sanitaria producida por la pandemia de la COVID-19, dado que a través de ellos se puede obtener información sobre cuál es la actuación de los perfiles sanitarios en un periodo de alto interés por la información sanitaria.

Este estudio se basa en la metodología AHP sobre la toma de decisiones. En línea con otras investigaciones, se ha cuantificado la información obtenida a través de la herramienta de acceso libre 
Twitonomy (Caldevilla-Domínguez et al., 2021, p. 3), procediendo a seleccionar las variables vinculadas a la difusión de mensajes en Twitter. Los datos extraídos corresponden a 63.985 tweets.

Para determinar las variables de estudio se ha acudido a dos expertos del ámbito de la comunicación de organismos públicos y privados. En la selección de los expertos se han tenido en cuenta aspectos como los años de experiencia en el ámbito de la comunicación corporativa; su trayectoria profesional en la comunicación en redes sociales y por último se ha tenido en cuenta el criterio de paridad. De este modo se han seleccionado dos personas expertas en comunicación corporativa, con más de 20 años de experiencia profesional vinculados a la comunicación estratégica. Uno de los expertos es Mercedes Pastor García, Dircom especializada en comunicación estratégica. Cuenta con una experiencia de más de 25 años en el ámbito de la comunicación y ha trabajado en materia de estrategia de comunicación en diferentes instituciones. Actualmente es directora de comunicación en el Ministerio de Sanidad. El otro experto es Cristóbal Fernández Muñoz, actual vicedecano de Comunicación y Relaciones Institucionales en la Facultad de Ciencias de la Información de la Universidad Complutense de Madrid (UCM). Tiene una experiencia de más de 20 años en el ámbito de la comunicación corporativa. Ha sido director de comunicación de la red social Tuenti y de Reclamaror.es, dirigiendo en ambos puestos la estrategia de comunicación de la entidad.

Estos criterios han sido contrastados con los utilizados en las investigaciones sobre la comunicación en Twitter de Guijarro et al. (2018, p. 1417); Lamirán-Palomares et al., (2020, p. 8). De este modo, se ha optado por seleccionar dos clústeres, el clúster 1: actividad de la cuenta; clúster 2: impacto de la cuenta, variando algunos de los criterios para ajustarlos más a las necesidades del estudio. De este modo, se ha optado por incluir el criterio eficacia, es decir, los retweets y favoritos recibidos de terceros respecto al número total de tweets realizados.

La tabla 3 muestra los 17 criterios analizados. Algunos de ellos son, el perfil de usuario de la cuenta, el número de seguidos; número de tweets totales; número de retweets que realiza la cuenta; el número de respuestas; la media tweets/día; el número de seguidores; la amplificación del mensaje a través del número de tweets retuiteados por otros usuarios, así como el número de veces que los tweets emitidos han sido retuiteados; el número de hashtags totales y las menciones hechas al perfil. 
Tabla 3. Variables de análisis

\begin{tabular}{|l|l|}
\hline \multicolumn{1}{|c|}{ Perfil de Twitter: } & \multicolumn{1}{c|}{ Análisis de los 3200 Tweets más recientes } \\
\hline Nivel & \multicolumn{1}{|c|}{ Nacional/regional } \\
\hline \multicolumn{2}{|c|}{ Clúster 1: Actividad de la cuenta } \\
\hline Idioma de publicación & \\
\hline Número de seguidos & \\
\hline Número de Tweets totales & \\
\hline Número de Retweets & \\
\hline Número de respuestas & \\
\hline Media Tweets/día & \\
\hline \multicolumn{2}{|c|}{ Clúster 2: Impacto de la cuenta } \\
\hline Número de seguidores & \\
\hline $\begin{array}{l}\text { Proporción de seguidores por persona tardía (mide el } \\
\text { nivel de influencia) }\end{array}$ & \\
\hline Número Retweets recibidos & \\
\hline Número de veces retuiteado por terceros & \\
\hline Media Retweets/Tweets & \\
\hline Número de favoritos recibidos & \\
\hline No veces marcado como favorito por terceros & \\
\hline Media favoritos/Tweets & \\
\hline Eficacia (Retweets de otros y favoritos de otros hacia él) / \\
Número total de Tweets
\end{tabular}

Fuente: elaboración propia a partir de Guijarro. 2018.

Definidas las variables, el siguiente paso es descomponer el problema y jerarquizarlo. Para poder comparar las alternativas utilizadas y los criterios es necesario determinar cuál de los 21 perfiles analizados realiza una mejor comunicación en Twitter en base a esos criterios.

En términos de calidad, cuando se elabora el Proceso de Análisis Jerárquico es necesario estudiar el nivel de consistencia del criterio de los expertos para que sea considerado válido, es decir, para que sea valorado como racional. De modo que para que el modelo planteado se considere válido, es imprescindible medir el índice de inconsistencia de la matriz que determina si el juicio de los expertos es aceptable o no (Favret et al., 2015, p. 6; Guijarro et al., 2018, p. 1420; LamiránPalomares et al., 2020, p. 7). Se considera consistente una razón de consistencia (RC) menor o igual de 0,10 (Velastegui et al., 2020, p. 163; Lamirán-Palomares et al., 2020, p. 8; Shan, 2021, p. 61). De este modo, previamente ha sido ponderada la importancia relativa de cada criterio por los expertos en comunicación teniendo en cuenta los niveles de consistencia necesarios para la aceptación del modelo AHP. 


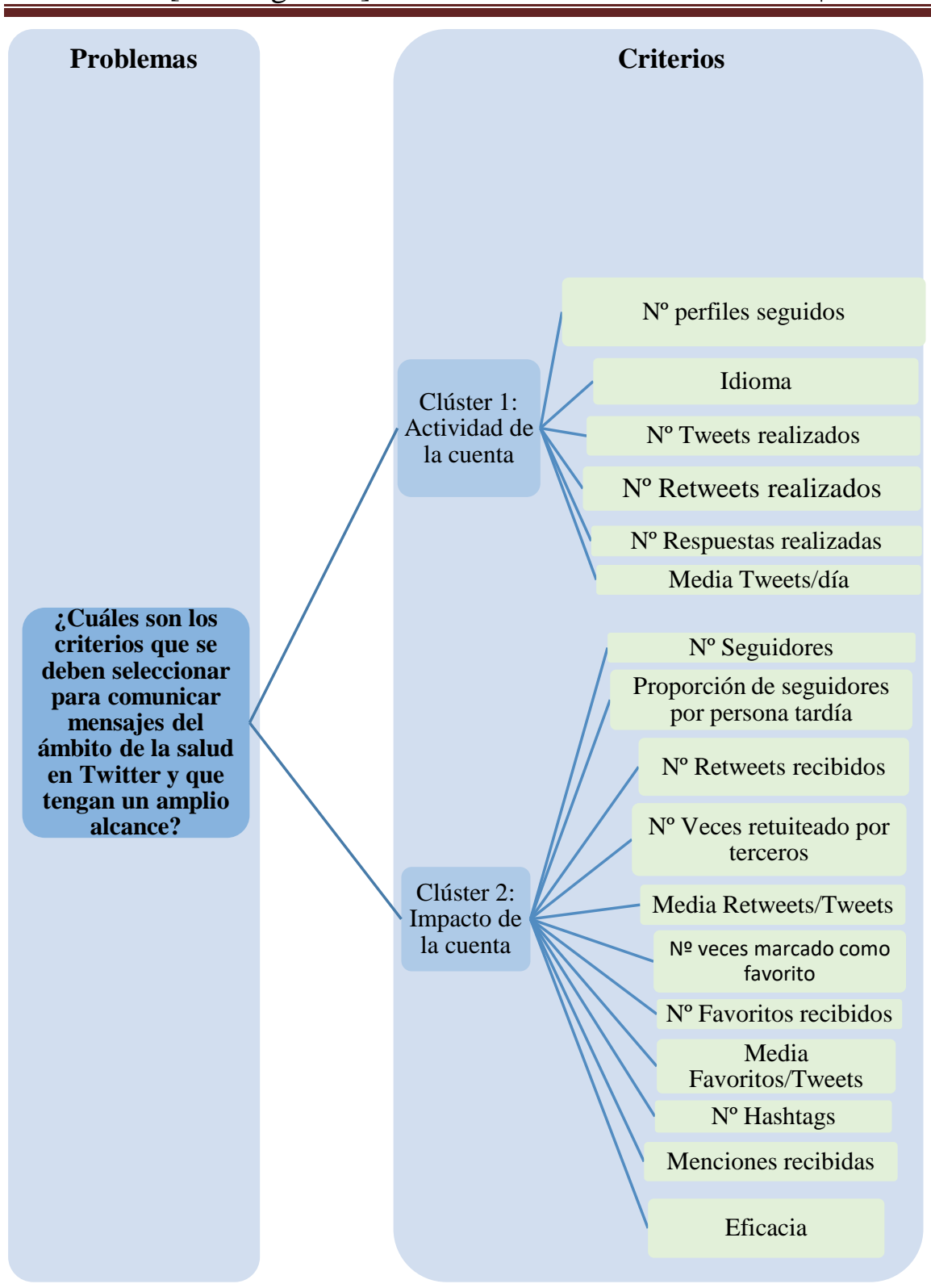

\begin{tabular}{|c|}
\hline Alternativas \\
\hline @ SanidadCLM \\
\hline @ibsalut \\
\hline @ riojasalud \\
\hline @ sanidadgob \\
\hline @ osakidetzaEJGV \\
\hline @icsca \\
\hline @snsalud_na \\
\hline @Murciasalud \\
\hline @Salud_JCYL \\
\hline @GVAsanitat \\
\hline @ saludand \\
\hline @SaludPublicaAra \\
\hline @ Ingesa_Ceuta \\
\hline @Ingesa_Melilla \\
\hline @ astursalud \\
\hline @SanidadeXunta \\
\hline @JuntaEx_Sanida \\
\hline @INGESAnidad \\
\hline @ SaludMadrid \\
\hline @SanidadGobCan \\
\hline @ saludcantabria \\
\hline
\end{tabular}

Figura 2: Jerarquización del problema Fuente: elaboración propia.

La figura 2 indica el problema que se pretende resolver, los 17 criterios que forman parte del proceso de decisión, condensados en dos clústeres. En el clúster 1 se engloban los criterios relacionados con la actividad de la cuenta y en clúster 2 los vinculados al impacto. Por último, se muestran los 21 perfiles de Twitter analizados, considerando cada uno de ellos una alternativa de comunicación.

Para determinar la importancia cualitativa de cada criterio se han realizado comparaciones pareadas utilizando la escala de comparación por pares establecida por Saaty (1980), aún en vigor (Beltrán et al., 2021, p. 252; Sirakaya et al., 2020, p. 5; Guijarro et al., 2018, pp. 1418-1419). Siguiendo la escala de 9 puntos de Saaty (1980), el resultado de la consulta a expertos ha dado lugar a dos matrices. En ellas se ha comparado la importancia relativa de cada criterio entre sí, en función a su dominancia. El resultado de la comparación por pares se puede observar en las matrices de las tablas 4 y 5 . 
RLCS, Revista Latina de Comunicación Social, 80, 89-117

[Investigación] DOI: 10.4185/RLCS-2022-1532| ISSN 1138-5820| Año 2022

Tabla 4. Matriz de comparaciones pareadas clúster 1: Actividad de la cuenta

\begin{tabular}{|l|l|l|c|c|c|c|}
\hline & Idioma & $\begin{array}{l}\mathrm{N}^{\circ} \\
\text { Seguidos }\end{array}$ & $\begin{array}{l}\mathrm{N}^{\circ} \begin{array}{c}\text { Tweets } \\
\text { realizados }\end{array} \\
\mathrm{N}^{\circ} \begin{array}{l}\text { Retweets } \\
\text { realizados }\end{array}\end{array}$ & $\begin{array}{l}\mathrm{N}^{\circ} \text { Respuestas } \\
\text { realizadas }\end{array}$ & $\begin{array}{l}\text { Media } \\
\text { Tweets/día }\end{array}$ \\
\hline Idioma & & 1 & $1 / 2$ & $1 / 2$ & $1 / 2$ & $1 / 2$ \\
\hline $\mathrm{N}^{\circ}$ Seguidos & & & $1 / 2$ & $2 / 1$ & $1 / 2$ & $1 / 2$ \\
\hline $\mathrm{N}^{\circ}$ Tweets realizados & & & & $2 / 1$ & 1 & 1 \\
\hline $\mathrm{N}^{\circ}$ Retweets realizados & & & & $1 / 2$ & $1 / 2$ \\
\hline $\begin{array}{l}\mathrm{N}^{\circ} \\
\text { realizadas }\end{array}$ & & & & & $1 / 2$ \\
\hline Media Tweets/día & & & & & & \\
\hline
\end{tabular}

Fuente: elaboración propia a través del criterio de dos expertos en comunicación Nota: Nivel de inconsistencia 0,03

Puede observarse que los expertos han considerado que el número de seguidos, de tweets realizados, de retweets, respuestas realizadas y la media de tweets/día tiene una dominancia moderada sobre el idioma.

Tabla 5. Matriz de comparaciones pareadas clúster 2: Impacto de la cuenta

\begin{tabular}{|c|c|c|c|c|c|c|c|c|c|c|c|}
\hline & 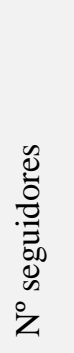 & 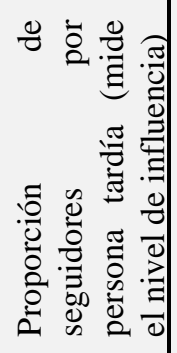 & 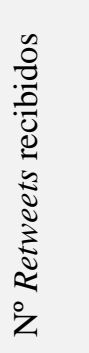 & 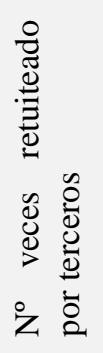 & 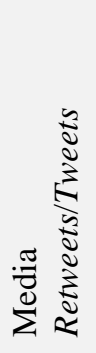 & 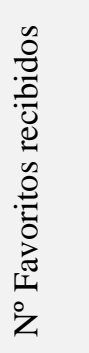 & 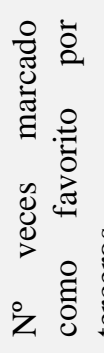 & 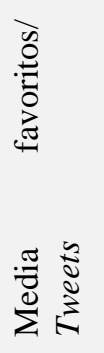 & 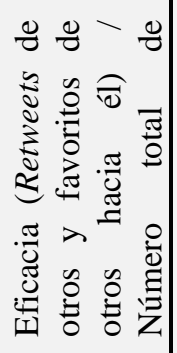 & $\begin{array}{l} \\
\\
0 \\
0 \\
\frac{\pi}{\pi} \\
\frac{\pi}{5} \\
\frac{7}{T} \\
0 \\
\text { Z }\end{array}$ & 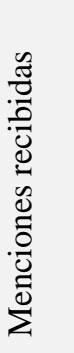 \\
\hline $\mathrm{N}^{\mathrm{o}}$ seguidores & & $1 / 2$ & $1 / 5$ & $1 / 2$ & $1 / 5$ & $1 / 2$ & $1 / 2$ & $1 / 2$ & $1 / 4$ & $2 / 1$ & $1 / 3$ \\
\hline $\begin{array}{l}\text { Proporción de } \\
\text { seguidores por } \\
\text { persona tardía } \\
\text { (mide el nivel } \\
\text { de influencia) }\end{array}$ & & & $1 / 5$ & $1 / 3$ & $1 / 3$ & $1 / 2$ & $1 / 2$ & $1 / 2$ & $1 / 4$ & $4 / 1$ & $1 / 3$ \\
\hline $\begin{array}{l}\mathrm{N}^{\mathbf{o}} \quad \text { Retweets } \\
\text { recibidos }\end{array}$ & & & & $1 / 2$ & 1 & $3 / 1$ & $3 / 1$ & $3 / 1$ & $1 / 2$ & $7 / 1$ & $2 / 1$ \\
\hline $\begin{array}{lr}\mathrm{N}^{\circ} & \text { veces } \\
\text { retuiteado } & \text { por } \\
\text { terceros } & \\
\end{array}$ & & & & & 1 & $3 / 1$ & $3 / 1$ & $4 / 1$ & 1 & $7 / 1$ & $2 / 1$ \\
\hline $\begin{array}{l}\text { Media } \\
\text { Retweets/Tweet } \\
s\end{array}$ & & & & & & $3 / 1$ & $3 / 1$ & $3 / 1$ & $1 / 3$ & $7 / 1$ & $2 / 1$ \\
\hline $\begin{array}{l}\mathrm{N}^{\mathrm{o}} \quad \text { Favoritos } \\
\text { recibidos }\end{array}$ & & & & & & & 1 & 1 & $1 / 3$ & $3 / 1$ & $1 / 2$ \\
\hline $\begin{array}{|rr|}\mathrm{N}^{\circ} & \text { veces } \\
\text { marcado } & \text { como } \\
\text { favorito } & \text { por } \\
\text { terceros } & \\
\end{array}$ & & & & & & & & 1 & $1 / 2$ & $3 / 1$ & $1 / 2$ \\
\hline $\begin{array}{l}\text { Media } \\
\text { favoritos/ } \\
\text { Tweets } \\
\end{array}$ & & & & & & & & & $1 / 2$ & $4 / 1$ & $1 / 2$ \\
\hline \begin{tabular}{|lr} 
Eficacia & \\
(Retweets & $\mathrm{de}$ \\
otros & $\mathrm{y}$ \\
favoritos & $\mathrm{de}$ \\
\end{tabular} & & & & & & & & & & $6 / 1$ & 1 \\
\hline
\end{tabular}




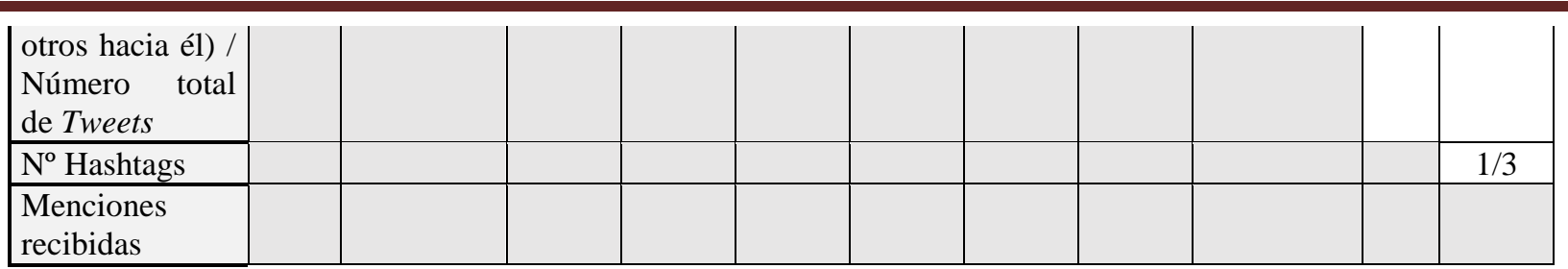

Fuente: elaboración propia a través del criterio de dos expertos en comunicación

Nota: Nivel de inconsistencia 0,03

Para determinar que la matriz de valoración de este estudio es aceptable, se ha medido la razón de inconsistencia calculada a través del programa Expert Choice. La razón de inconsistencia (RI) para las matrices representadas en las tablas 4 y 5 es de 0,03 . Al ser una RI menor de 0,1 , entra dentro de los parámetros aceptados (Mendoza et al., 2019, p. 352).

El paso final es conocer los vectores propios y ponderar cada una de las alternativas para conocer cuál es la variable que explica el problema en función de la valoración inicial que realizaron los expertos (Guijarro et al., 2018, p. 1420).

\section{Resultados}

Cada uno de los perfiles analizados puede mostrar un mayor o menor desarrollo de su estrategia comunicativa en Twitter en función del tiempo que lleven utilizando la aplicación. A través del Proceso de Análisis Jerárquico se obtiene información acerca de cuál de las 21 alternativas de comunicación analizadas es la más adecuada para difundir información en materia de salud en la red social Twitter y conocer el peso ponderado de cada criterio (tabla 6). Tras el tratamiento de la matriz de información aportada por los expertos consultados se observa que, de entre los dos clústeres, le otorgan una mayor relevancia al clúster 2: Impacto de la cuenta, con un peso de 0,750 , mientras que el clúster 1: actividad de la cuenta tiene un peso de 0,250 .

Tabla 6. Peso ponderado de los clústeres

\begin{tabular}{|c|c|}
\hline Clúster & Peso del clúster \\
\hline C1. Actividad de la cuenta & 0,250 \\
\hline C2. Impacto de la cuenta & 0,750 \\
\hline
\end{tabular}

Fuente: elaboración propia

El peso de cada uno de los criterios se observa en la tabla 7. Dentro del Clúster 1: Actividad de la cuenta, el criterio media de tweets por día es el que mayor relevancia presenta con un peso del $24,8 \%$, seguido de los tweets realizados $(21,6 \%)$ y del número de respuestas realizadas $(19,6 \%)$. Con estos datos se puede indicar que en las estrategias de comunicación en redes sociales digitales la constancia es un factor relevante, confirmando las observaciones llevadas a cabo por Guijarro et al. (2018).

En el clúster 2: Impacto de la cuenta, el criterio más importante es la eficacia $(17,3 \%)$, seguido del número de veces que ha sido retuiteado por terceros $(16,4 \%)$ y el número de retweets $(14,8 \%)$. 
Tabla 7. Peso de cada uno de los criterios. Año 2020

\begin{tabular}{|l|c|c|c|}
\hline \multicolumn{1}{|c|}{$\begin{array}{c}\text { Porcentaje de } \\
\text { peso relativo } \\
\text { según clúster }\end{array}$} & Peso global & $\begin{array}{c}\text { Porcentaje } \\
\text { peso global }\end{array}$ \\
\hline \multicolumn{2}{|c|}{ Clúster 1: Actividad de la cuenta } \\
\hline Idioma & 10 & 0,025 & 2,5 \\
\hline № Seguidos & 12,8 & 0,032 & 3,2 \\
\hline № Tweets realizados & 21,6 & 0,054 & 5,4 \\
\hline № Retweets realizados & 11,2 & 0,028 & 2,8 \\
\hline № Respuestas realizadas & 19,6 & 0,049 & 4,9 \\
\hline Media Tweets/día & 24,8 & 0,062 & 6,2 \\
\hline \multicolumn{1}{|c|}{ Clúster 2: Impacto de la cuenta } & \\
\hline № seguidores & 3,47 & 0,026 & 2,6 \\
\hline $\begin{array}{l}\text { Proporción de seguidores por persona tardía } \\
\text { (mide el nivel de influencia) }\end{array}$ & 4,27 & 0,032 & 3,2 \\
\hline № Retweets recibidos & 14,80 & 0,111 & 11,1 \\
\hline № veces retuiteado por terceros & 16,40 & 0,123 & 12,3 \\
\hline Media Retweets/Tweets & 14,53 & 0,109 & 10,9 \\
\hline № Favoritos recibidos & 5,60 & 0,042 & 4,2 \\
\hline № veces marcado como favorito por terceros & 5,87 & 0,044 & 4,4 \\
\hline Media favoritos/Tweets & 5,87 & 0,044 & 4,4 \\
\hline $\begin{array}{l}\text { Eficacia (Retweets de otros y favoritos de } \\
\text { otros hacia él) / Número total de Tweets }\end{array}$ & 17,33 & 0,130 & 13 \\
\hline № Hashtags & 2,00 & 0,015 & 1,5 \\
\hline Menciones recibidas & 9,87 & 0,074 & 7,4 \\
\hline
\end{tabular}

Fuente: elaboración propia

Conocido el peso de cada criterio, en el gráfico 1 se muestra la ponderación de cada uno de los 21 perfiles analizados. En primer lugar, se sitúa el perfil del Ministerio de Sanidad, Consumo y Bienestar Social: Min. Sanidad (MSCBS), @ sanidadgob, con una importancia según el modelo de un 10,7\%, prácticamente igualado con el perfil del Institut Català de la Salut. Departament de Salut. Generalitat de Catalunya, @icscat, con un 10,6\%. En tercer lugar, se encuentra Salud Castilla y León, @Salud_JCYL, con un peso de 9,1\% respecto al total. 
RLCS, Revista Latina de Comunicación Social, 80, 89-117

[Investigación] DOI: 10.4185/RLCS-2022-1532| ISSN 1138-5820| Año 2022

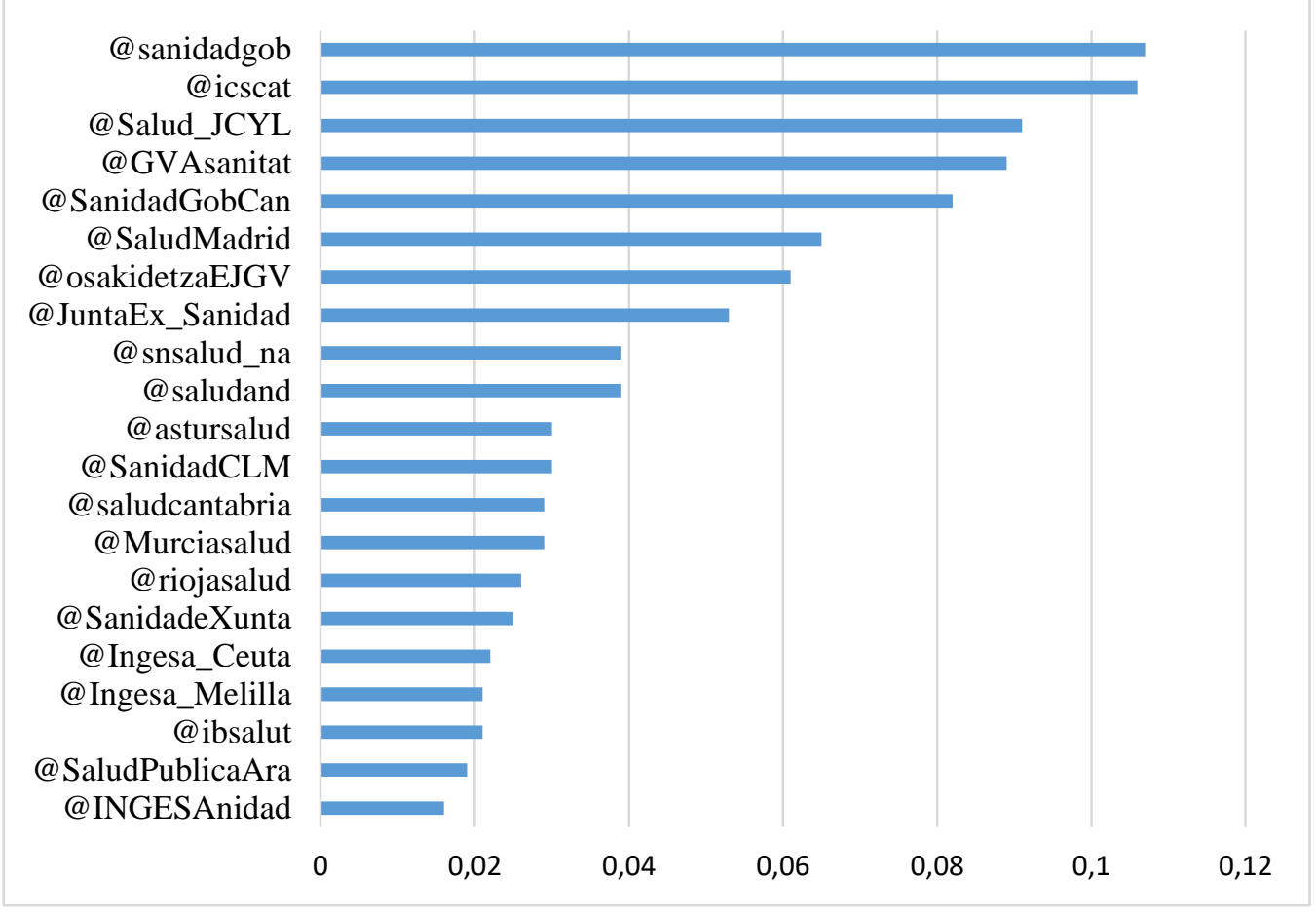

Gráfico 1: Peso ponderado de cada alternativa.

Fuente: Elaboración propia

En el gráfico 2 se muestra el peso de las 21 alternativas desglosado por cada uno de los dos clústeres. En su elaboración se ha tenido en cuenta el peso de las 17 variables que componen los clústeres actividad de la cuenta e impacto.

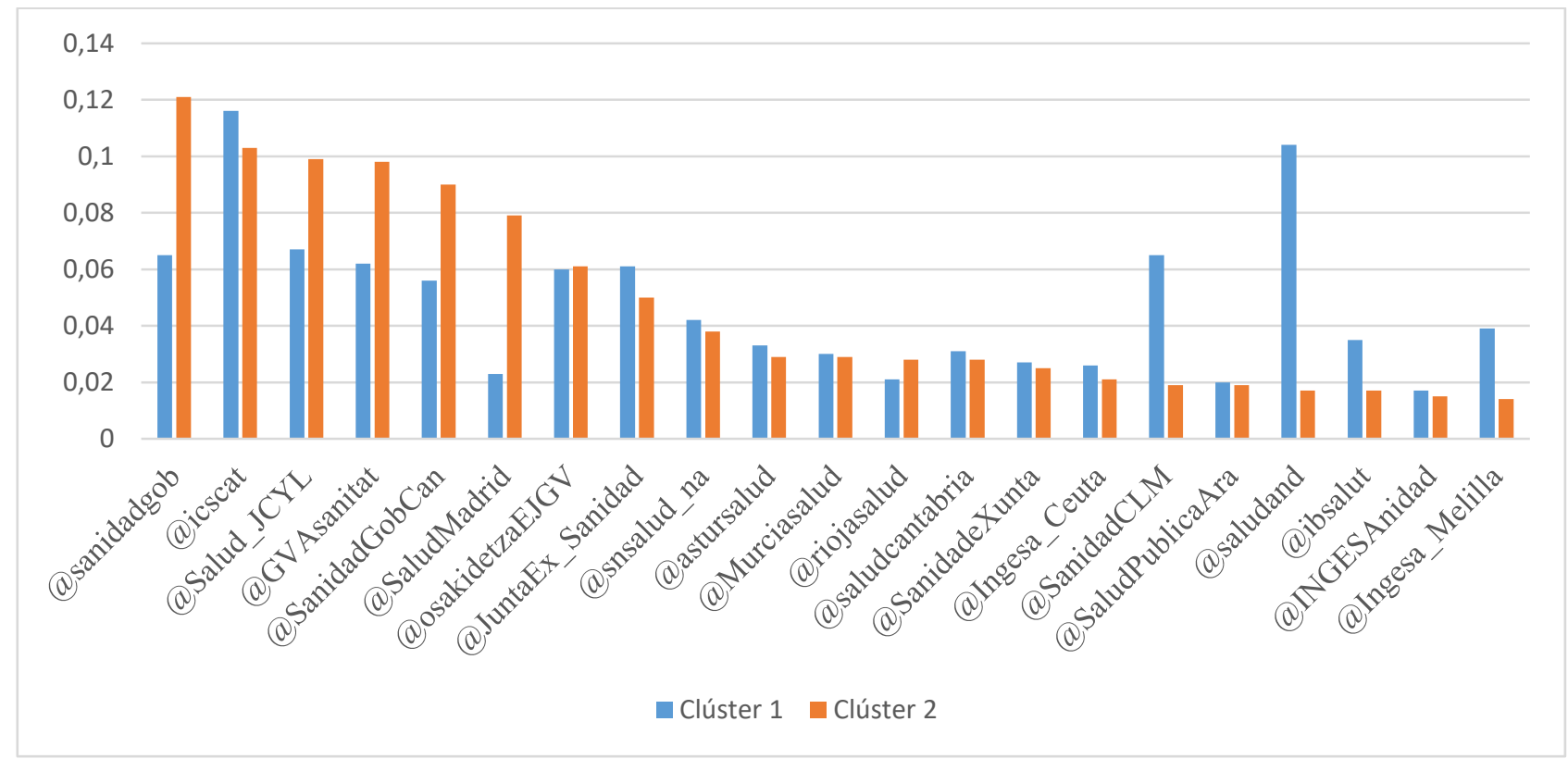

Gráfico 2: Peso de las alternativas respecto al Clúster 1: Actividad de la cuenta y Clúster 2: Impacto de la cuenta

Fuente: elaboración propia 
Esta información muestra, según los criterios valorados, cuáles son los perfiles más relevantes. Asimismo, los datos corroboran que el mayor peso del modelo recae sobre el clúster 2.

La alternativa del Ministerio de Sanidad, Consumo y Bienestar Social: Min. Sanidad (MSCBS), @sanidadgob que, como se ha comentado anteriormente es la que mayor relevancia tiene en el modelo, junto a @icscat, es a su vez, la que mayor peso tiene en el clúster impacto. Se observa también que el Institut Català de la Salut. Departament de Salut. Generalitat de Catalunya, @icscat, tiene un perfil más equilibrado entre ambos clústeres. Es llamativo el caso del perfil de la Consejería de Salud y Familias de la Junta de Andalucía, @saludand. Analizando el peso del modelo de este perfil, se observa que se sitúa en la novena posición respecto a los 21 perfiles institucionales analizados, sin embargo, ha alcanzado esa posición, siendo uno de los perfiles con menor peso en el clúster impacto (clúster de mayor peso en el modelo), pero contrarrestando esta situación con un elevado peso en el clúster actividad $(10,4 \%)$ que provoca que ocupe la segunda posición junto al perfil del instituto catalán $(11,4 \%)$ dentro de ese clúster.

\section{Discusión y conclusiones}

Al establecer una estrategia de comunicación en redes sociales digitales, los responsables de los perfiles institucionales de ámbito sanitario en Twitter deben tener presente diferentes criterios que dificultan la toma de decisión al difundir información en la red.

Este estudio proporciona un análisis de la comunicación realizada en Twitter por 21 organismos públicos vinculados al ámbito sanitario, en los que se ha observado la relación existente entre los distintos elementos que componen la comunicación en esta red social y el alcance de la información publicada en ella. Para ello, se ha elaborado un Analytic Hierarchy Process (AHP) o Proceso de Análisis Jerárquico que facilita el conocimiento de la estrategia de comunicación en salud que realizan en Twitter cada uno de estos 21 organismos públicos. A través de él, este estudio propone un modelo multicriterio que tiene como finalidad posibilitar la toma de decisión en el desarrollo de la comunicación en Twitter, en línea con la idea de estudios previos (Guijarro et al., 2018).

Este trabajo facilita conocer aquellos criterios que benefician la comunicación en Twitter y que pueden ser tenidos en cuenta por distintos organismos cuando realicen estrategias de comunicación digital, en consonancia con la tesis de autores que señalan la importancia que tiene conocer los aspectos clave que determinan que una comunicación tenga un mayor alcance, propicie una mayor interacción e intercambio de información (Sanz-Lorente et al., 2018, p. 5; Thackeray et al., 2012, p. $5)$.

Los datos confirman que el clúster impacto es el de mayor peso en la comunicación en Twitter. En este estudio, se ha incluido además el criterio eficacia, entendida como el número de retweets y favoritos recibidos sobre número total de tweets, observándose que es uno de los más relevantes dentro del clúster impacto de la cuenta.

Se ratifica que, en las estrategias de comunicación en redes digitales, si se observa el impacto de un perfil de Twitter, entre los tres elementos más relevantes se encuentran, por este orden, la eficacia (13\%); el número de veces retuiteado por terceros $(12,3 \%)$ y el número de retweets recibidos $(11 \%)$ en línea con los resultados obtenidos en otros ámbitos como el de seguridad (Guijarro et al., 2018, p. 1422). Analizando los factores relacionados con la actividad del perfil destaca la media de tweets al día, con un $24 \%$ respecto al peso de ese clúster. 
Los resultados de este estudio revelan que el organismo que obtiene un mayor peso en el modelo propuesto es el correspondiente al Ministerio de Sanidad, Consumo y Bienestar Social: Min. Sanidad (MSCBS), @sanidadgob, prácticamente igualado con la alternativa del Institut Català de la Salut. Departament de Salut. Generalitat de Catalunya, a pesar de que uno de los aspectos que dificultan este posicionamiento es el idioma, dado que utiliza exclusivamente la lengua catalana, a diferencia de otras cuentas regionales que han combinado otros idiomas junto al Castellano. Este hecho hace que su difusión se restrinja entre los usuarios de habla catalana y por ello, la cobertura pueda ser menor.

Una de las conclusiones que se puede extraer de este estudio es que, con independencia del tipo de organización que se analice, el AHP es una herramienta facilitadora de la toma de decisiones en el ámbito de la comunicación digital en redes sociales (Osorio y Orejuela, 2008, p. 252; Guijarro et al., 2018, p. 1424).

Cabe señalar algunas limitaciones presentes en este estudio. Debido a lo novedoso que es el uso del AHP en el ámbito de la comunicación y las redes sociales, existe espacio para refinar la metodología empleada. En este estudio solo se han tenido en cuenta elementos vinculados a la actividad de la cuenta y a su impacto. Se pueden incluir algunos aspectos relevantes como son el análisis del contexto social y tecnológico en el que actúa cada perfil de Twitter analizado, así como las variables relacionadas con la organización como la existencia de un plan de comunicación o un departamento encargado de la comunicación en redes sociales. Asimismo, presenta limitaciones respecto a la recogida de datos, ya que la aplicación Twitonomy ofrece resultados limitados, reduciendo la información a los últimos 3.200 Tweets de cada perfil.

Como futuras líneas de investigación se plantea la posibilidad de analizar cuál ha sido el impacto de la crisis sanitaria producida por la COVID-19 en la comunicación digital de la salud, elaborando una comparativa del peso de estas alternativas antes y después de que se produjera la pandemia. Asimismo, se considera relevante complementar esta investigación con el análisis de contenido de los tweets seleccionados para observar su impacto en el modelo. También, se sugiere la elaboración de entrevistas en profundidad a los responsables de comunicación de los perfiles analizados para conocer sus criterios, preferencias y valores a la hora de establecer esa comunicación y las dificultades a las que se ven sometidos/as en la toma de decisión.

\section{Referencias}

Agwa-Ejon, J. F., Mulaba-Bafubiandi, A. y Pretorius, J. H. (2017). The multi-criteria decision making, a tool for the promotion of technological inter-connected world through digital decision making. In 2017 Portland International Conference on Management of Engineering and Technology (pp. 1-7). https://ieeexplore.ieee.org/abstract/document/8125301/authors\#authors

Barajas-Galindo, D. E. y Rodríguez Carnero, M. G. (2020). Scientific Dissemination in the Twitter Era. Endocrinología, Diabetes y Nutrición (English ed.) 67(5), 295-296. https://doi.org/10.1016/j.endien.2020.03.003

Bartlett, C. y Wurtz, R. (2015). Twitter and Public Health. Journal of Public Health Management and Practice 21(4), 375-383 https://doi.org/10.1097/PHH.0000000000000041 
Beltrán, J. M., Acurio, G. F. y Alulema, P. S. (2021). Método AHP de Saaty para determinar los factores del quantum indemnizatorio por daño inmaterial en materia penal en Ecuador. Universidad y Sociedad, 13(2), 249-256. https://rus.ucf.edu.cu/index.php/rus/article/view/1962

Cabrera, S. y Clavijo, M. (2020). Discurso científico en Twitter en el primer trimestre de pandemia en $\quad$ Ecuador. $\quad$ \#PerDebate, $4,128-155$. https://revistas.usfq.edu.ec/index.php/perdebate/article/view/1882

Caldevilla-Domínguez, D., Rodríguez Terceño, J. y Barrientos-Báez, A. (2019). El malestar social a través de las nuevas tecnologías: Twitter como herramienta política. Revista Latina de Comunicación Social, 74, 1264-1290. http://www.revistalatinacs.org/074paper/1383/66es.html

Caldevilla-Domínguez, D., Barrientos-Báez, A. y Padilla-Castillo, G. (2021). COVID-19 as a Trigger for Sustainable Tourism and Eco-Influencers on Twitter. Sostenibilidad 2021, 13. https://www.preprints.org/manuscript/202101.0636/v1

Centers for Disease Control and Prevention. (2011). The health communicator's social media toolkit. Atlanta, GA: Centers for Disease Control and Prevention.

https://www.cdc.gov/healthcommunication/ToolsTemplates/SocialMediaToolkit_BM.pdf

Eurostat. (2020). Personas que utilizan Internet para buscar información relacionada con la salud. Navegador de datos. https://ec.europa.eu/eurostat/databrowser/view/tin00101/default/table?lang=en

Eysenbach, G. (2011). Infodemiology and infoveillance: Tracking Online Health Information and Cyberbehavior for Public Health. American Journal of Preventive Medicine, 40(5, S2), S154S158. https://doi.org/10.1016/j.amepre.2011.02.006

Eysenbach, G. (2020). How to fight an infodemic: the four pillars of infodemic management. Journal of medical internet research, 22(6) https://doi.org/10.2196/21820

Favret, F., Rodríguez, F. M. y Labat, M. D. (2015). Regresión con SVM para reducir la inconsistencia de la matriz AHP. In Argentine Symposium on Artificial Intelligence (ASAI 2015)JAIIO, 44. https://cutt.ly/fbJuInF

Ferrer-Serrano, M., Latorre-Martínez, M. P. y Lozano-Blasco, R. (2020). Universidades y comunicación. Papel de Twitter durante el inicio de la crisis sanitaria de la Covid-19. Profesional de la información, 29(6), 1-18. https://doi.org/10.3145/epi.2020.nov.12

Guijarro, E., Santandreu Mascarell, C., Canós-Darós, L., Díez-Somavilla, R. y Babiloni, E. (2018). Técnicas multicriterio aplicadas a las redes sociales en el sector público: el uso de Twitter por distintos perfiles de fuerzas y cuerpos de seguridad. Revista Latina de Comunicación Social, 73, 1412-1427. http://www.revistalatinacs.org/073paper/1314/73es.html

González, N. (2019). AHP: un método para fortalecer la toma de decisiones en SST. PrevenBlog. El Blog de PrevenControl. https://cutt.ly/KbJiQhG

Guo, J. W., Sisler, S. M., Wang, C. Y. y Wallace, A. S. (2021). Exploring experiences of COVID19-positive individuals from social media posts. Int $J$ Nurs Pract. https://doi.org/10.1111/ijn.12986 
Hou, Z. Y., Tong, Y.X., Du, F. X., Lu, L.Y., Zhao, S. H., Yu, K. X., Piatek, S. J., Larson, H. J. y Lin, L. E. S. (2021). Assessing COVID-19 Vaccine Hesitancy, Confidence, and Public Engagement: A Global Social Listening Study. J Med Internet Res, 23(6) https://preprints.jmir.org/preprint/27632

Hurtado, T. y Bruno, G. (2005). El Proceso de Análisis Jerárquico (AHP) como Herramienta para la Toma de Decisiones en la Selección de Proveedores. [Tesis de Licenciado en Investigación Operativa. Facultad de ciencias Matemáticas. UNMSM. Lima, Perú] http://sisbib.unmsm.edu.pe/bibvirtualdata/Tesis/Basic/toskano_hg/toskano_hg.pdf

Jordan, S. E., Hovet, S. E., Fung, I. C. H., Liang, H., Fu, K.W. y Tse, Z.T.H. (2018). Using twitter for public health surveillance from monitoring and prediction to public response. Data. 4(6), 1-20. https://doi.org/10.3390/data4010006

Lamirán-Palomares, J. M, Baviera, T. y Baviera-Puig, A. (2020). Influencers deportivos en Twitter. Análisis y estudio comparativo de los mundiales de ciclismo en pista 2016 y 2018. Ciencias Sociales, 9(10), 169. MDPI AG. http://dx.doi.org/10.3390/socsci9100169

Ley 12/2001 de 21 de diciembre, de Ordenación Sanitaria de la Comunidad de Madrid. https://cutt.ly/6bJiSK3

Manfredi-Sánchez, J., Amado-Suárez, A. y Waisbord, S. (2021). Presidential Twitter in the face of COVID-19: Between populism and pop politics. Comunicar, 66, 83-94. https://doi.org/10.3916/C66-2021-07

Medina, P. (2018). El rol de las redes sociales en la comunicación de marca de los hospitales españoles. adComunica. Revista Científica de Estrategias, Tendencias e Innovación en Comunicación, 15, 215-233. http://dx.doi.org/10.6035/2174-0992.2018.15.11

Mendoza, A., Solano, C., Palencia, D. y García, D. (2019). Aplicación del proceso de jerarquía analítica (AHP) para la toma de decisión con juicios de expertos. Ingeniare. Revista chilena de ingeniería, 27(3), 348-360. https://dx.doi.org/10.4067/S0718-33052019000300348

Myrick, J., Holton, A., Himboim, I. y Love, B. (2016). Stupidcancer: Exploring a Typology of Social Support and the Role of Emotional Expression in a Social Media Community. Health Communication, 31(5), 596-605. http://dx.doi.org/10.1080/10410236.2014.981664.

Navas-Martín, M. A., Albornos-Muñoz, L. y Escandell-García, C. (2012). Acceso a fuentes de información sobre salud en España: cómo combatir la infoxicación. Enfermería Clínica, 22(3), 154-158. https://doi.org/10.1016/j.enfcli.2012.04.001

OMS. (2020). Primera Conferencia de la OMS sobre Infodemiología. Centro de Prensa. https://cutt.ly/PbJiVfX

ONTSI. (2020). Individuos que usan Internet para buscar información relacionada con la salud. Indicadores. https://cutt.ly/nbJiMWB

Osorio, J. C. y Orejuela, J. P. (2008). El proceso de análisis jerárquico (AHP) y la toma de decisiones multicriterio. Ejemplo de aplicación. Scientia et technica, 2(39), 247-252. https://revistas.utp.edu.co/index.php/revistaciencia/article/view/3217 
Paredes, J. A. (2010). Toma de decisiones. Proceso Analítico Jerárquico aplicado a la selección de herramienta de educación virtual. FPUNE Scientific, 6.

Park, H., Reber, B. H. y Chon, M. G. (2015). Tweeting as Health Communication: Health Organizations' Use of Twitter for Health Promotion and Public Engagement. Journal of health communication, 21(2), 188-198. https://doi.org/10.1080/10810730.2015.1058435

Park, H., Rodgers, S. y Stemmle, J. (2013). Analyzing Health Organizations' Use of Twitter for Promoting Health Literacy. Journal of Health Communication: International Perspectives, 18(4), 410-425. https://doi.org/10.1080/10810730.2012.727956

Pérez, P. L., Berná, C. B. y Arroyas, E. (2013). La interpretación simbólica de los desahucios en España a través del frame: un análisis semántico de la conversación en Twitter. In I Congreso Internacional de Comunicación y Sociedad Digital (p. 5). UNIR-Universidad Internacional de La Rioja. https://dialnet.unirioja.es/servlet/articulo?codigo=4247767

Pisano González, M. M., Prieto Rodríguez, M. A., García Regidor, M. C., Iglesias Alonso, F., Ochoa de Retana García, L., López Fernández, D., Aguas Torres, A., Carles Mach, J., Peñacoba Maestre, D., Vázquez Álvarez, R., Currás Mateo, D., Moreno Sancho, M.L., Cáceres Teijeiro, Y., García Cernuda, V., Amil Buján, P., Salamanca Bautista, P., Monteagudo Romero, J., Mateos Rodilla, J., Ferrer Arnedo, C., ... Gómez i Verdeguer, X. (2021). Aportación de las Escuelas de Salud y Programas de Autogestión durante la pandemia COVID-19. Metas Enferm, 24(3), 3-6. https://doi.org/10.35667/MetasEnf.2021.24.1003081727

Pulido-Polo, M., Hernández-Santaolalla, V. y Lozano-González, A. A. (2021). Uso institucional de Twitter para combatir la infodemia causada por la crisis sanitaria de la Covid-19. Profesional de la información, 30(1), 1-15. https://doi.org/10.3145/epi.2021.ene.19

Rando, D. y de las Heras, C. (2016). Análisis de la comunicación corporativa de los hospitales andaluces vía twitter. Opción: Revista de Ciencias Humanas y Sociales, 32, 557-576. https://produccioncientificaluz.org/index.php/opcion/article/view/21552

Sanz-Lorente, M., Wanden-Berghe, C., Castejón-Bolea, R. y Sanz-Valero, J. (2018). Web 2.0 Tools in the Prevention of Curable Sexually Transmitted Diseases: Scoping Review. J Med Internet Res, 20(3), e113.https://doi.org/10.2196/jmir.8871

Saaty, T. L. (1980). The Analytic Hierarchy Process: Planning, Priority Setting, Resource Allocation, McGraw-Hill.

Schillinger, D., Chittamuru, D. y Ramírez, A. S. (2020). From 'infodemics' to health promotion: a novel framework for the role of social media in public health. American journal of public health, 110(9), 1393-1396. https://doi.org/10.2105/AJPH.2020.305746

Sendra-Toset, A., y Farré-Coma, J. (2016). Las instituciones de salud pública y las redes sociales: una revisión sistemática. Revista Española de Comunicación en Salud, 285-299. http://dx.doi.org/10.20318/recs.2016.3452 
Sinnenberg, L., Buttenheim, A. M., Padrez. K., Mancheno, C., Ungar, L. y Merchant, R. M. (2017). Twitter as a Tool for Health Research: A Systematic Review. AJPH Research, 107(1), e1-e8. https://ajph.aphapublications.org/doi/10.2105/AJPH.2016.303512

Sirakaya, A., De Brucker, K. y Vanagt, T. (2020). Designing regulatory frameworks for access to genetic resources: A multi-stakeholder multi-criteria approach. Frontiers in Genetics, 11, 1-22. https://doi.org/10.3389/fgene.2020.549836

Shan, D. (2021). La construcción de un modelo profesional de profesores de educación física en colegios y universidades desde la perspectiva de servicio de salud pública. Revista Brasileira de Medicina do Esporte, 27(spe), 59-61. https://doi.org/10.1590/1517-8692202127012020_0093

Thackeray, R., Neiger, B. L., Smith, A. K. y Van Wagenen, S. B. (2012). Adoption and use of social media among public health departments. BMC public health, 12(242), 1-6. https://doi.org/10.1186/1471-2458-12-242

Velastegui, R., Rosero, C., Sánchez, C. y Reyes, J. (2020). Evaluación de alternativas para uso de recursos públicos en emergencias mayores usando proceso jerárquico analítico (AHP): Caso de estudio cantón de Ambato. Revista Ibérica De Sistemas e Tecnologias De Informação (RISTI), E25,159-172. http://www.risti.xyz/issues/ristie25.pdf

Wang, Y., Hao, H. y Platt, L. S. (2021). Examining risk and crisis communications of government agencies and stakeholders during early-stages of COVID-19 on Twitter, Computers in Human Behavior, 114, https://doi.org/10.1016/j.chb.2020.106568.

Xifra, J. (2020). Comunicación corporativa, relaciones públicas y gestión del riesgo reputacional en tiempos del Covid-19. El Profesional de la Información, 29(2), 1-18. https://doi.org/10.3145/epi.2020.mar.20

Yepes, V. (2018). Proceso Analítico Jerárquico (Analytic Hierarchy Process, AHP). victoryepes.blogs. https://victoryepes.blogs.upv.es/2018/11/27/proceso-analitico-jerarquico-ahp/

Zolbanin, H. M., Zadeh, A. H. y Davazdahemami, B. (2021). Miscommunication in the age of communication: A crowdsourcing framework for symptom surveillance at the time of pandemics. International journal of medical informatics, $151, \quad$ 1-7. https://doi.org/10.1016/j.ijmedinf.2021.104486 


\title{
AUTORES:
}

\section{Eva Matarín Rodríguez-Peral}

Socióloga y doctora en Comunicación Audiovisual, Publicidad y Relaciones Públicas. Es profesora del área de Sociología en la Universidad Rey Juan Carlos; profesora en el Máster Universitario en Educación Inclusiva e Intercultural de la Universidad Internacional de la Rioja. Está acreditada por Agencia Nacional de Evaluación de la Calidad y Acreditación (ANECA) en la figura de Profesor Ayudante Doctor (PAD). Es miembro de la Asociación de Investigación Científica Icono 14. Ha publicado artículos en revistas científicas indexadas y capítulos de libro en editoriales como Tirant Lo Blanch; McGraw-Hill y Catarata. Sus líneas de investigación abordan el estudio de las migraciones, la educación y la salud.

\section{Índice H: 2}

Orcid ID: https://orcid.org/0000-0002-1701-3911

Google Scholar: https://scholar.google.es/citations?user=Z77V01cAAAAJ\&hl=es\&oi=ao

ResearchGate: https://www.researchgate.net/profile/Eva-Matarin-Rodriguez-Peral

Scopus ID: https://www.scopus.com/authid/detail.uri?authorId=57209718289

Academia.edu: https://urjc.academia.edu/EvaMatarín

\section{Tomás Gómez Franco}

Doctor en Economía aplicada, licenciado en CC Económicas y Empresariales y graduado en Derecho. Profesor en la Universidad Francisco de Vitoria, profesor de Economía en la universidad Carlos III de Madrid y profesor invitado en Saint Kolbe University del estado de Florida (EEUU). Autor de publicaciones en el campo de Economía de la Salud. Tuvo diferentes responsabilidades políticas entre las que cabe citar: alcalde de la Villa de Parla, diputado portavoz en la Asamblea de Madrid y senador del estado.

\section{Índice H: 1}

Orcid ID: https://orcid.org/0000-0001-6603-7824

Google Scholar: https://scholar.google.es/citations?user=5lMN7mcAAAAJ\&hl=es\&oi=ao

ResearchGate: https://www.researchgate.net/profile/Tomas_Gomez10

Scopus ID: https://www.scopus.com/authid/detail.uri?authorId=57219835439

Academia.edu: https://uc3m.academia.edu/TOMASGOMEZFRANCO

\begin{abstract}
Almudena García Manso
Doctora y licenciada en Sociología; Máster en Sociedad de la Información (2001). Postdoctorado estancia e investigación- en Derechos Humanos y Violencia contra la mujer (Universidade Federal do Maranhão); Postdoctorado-estancia e investigación- Salud Pública Global (Universidad de Fortaleza). PCD: Universidad Rey Juan Carlos (2009). PAD y PCD: Universidad Alfonso X el Sabio (2000-2007). Profesora Invitada Máster de Bioética (Universidad Militar Nueva Granada 2019-2021); Profesora Invitada colaboradora Máster en Gerontología (Universidade Federal do Paraíba 20192021); Profesora Máster en Derechos Humanos (Universidade Federal do Maranhao). Líneas de investigación: methaodos.org grupo de investigación URJC; Grupo de Investigación Red Temática Internacional en Hermenéutica Socio Ambiental y Formación Humana de Investigación, Universidad Autónoma del Estado de México; Observatorio Iberoamericano de Salud y Ciudadanía (Instituto de Salud Pública de la Universidad de Oporto); Grupo de investigación Medicina Social: Derecho, Salud y Ciudadanía (Universidade Federal do Paraíba)

Índice H: 12

Orcid ID: https://orcid.org/0000-0002-8781-5020

Google Scholar: https://scholar.google.es/citations?user=k1myhqcAAAAJ\&hl=es\&oi=ao

Scopus ID: https://www.scopus.com/authid/detail.uri?authorId=56041646500
\end{abstract}


RLCS, Revista Latina de Comunicación Social, 80, 89-117

[Investigación] DOI: 10.4185/RLCS-2022-1532| ISSN 1138-5820| Año 2022

\section{ANEXO I}

Tabla 8. Datos de los 21 perfiles de Twitter analizados

\begin{tabular}{|c|c|c|c|c|c|c|c|}
\hline \multicolumn{8}{|c|}{ ACTIVIDAD DE LA CUENTA } \\
\hline & $\begin{array}{l}\text { Perfil: } \\
\text { @sanidadexunta }\end{array}$ & $\begin{array}{l}\text { Perfil: } \\
\text { @SaludCantabria }\end{array}$ & $\begin{array}{l}\text { Perfil: } \\
\text { @sanidadgob }\end{array}$ & $\begin{array}{l}\text { Perfil: } \\
\text { @INGESAnidad }\end{array}$ & \begin{tabular}{|l} 
Perfil: \\
@SanidadCLM
\end{tabular} & $\begin{array}{l}\text { Perfil: } \\
\text { @Salud_JCYL }\end{array}$ & $\begin{array}{l}\text { Perfil: } \\
\text { @GVAsanitat }\end{array}$ \\
\hline Análisis & $\begin{array}{l}3.20020 c t 2019 \\
\text { (may 21) }\end{array}$ & $\begin{array}{l}3,10511 \text { de febrero } \\
2020\end{array}$ & $\begin{array}{l}320014 \text { Marzo } \\
2020-27 \text { Nov } \\
2020\end{array}$ & $\begin{array}{l}11275 \text { Noviembre } \\
2014-06 \text { Diciembre } \\
2020\end{array}$ & $\begin{array}{l}318411 \text { Mayo } \\
2018-06 \\
\text { Diciembre } 2020\end{array}$ & $\begin{array}{l}320003 \text { Marzo } \\
2020-06 \\
\text { Diciembre } \\
2020\end{array}$ & $\begin{array}{l}319921 \text { Junio } \\
2019-06 \\
\text { Diciembre } \\
2020\end{array}$ \\
\hline Nivel & Regional & Regional & Nacional & Nacional & Regional & Regional & Regional \\
\hline \begin{tabular}{|l} 
Idioma de \\
publicación
\end{tabular} & Gallego y español & Español & Español & Español & Español & Español & Español \\
\hline \begin{tabular}{|l|} 
Año en el que \\
se unió a \\
Twitter
\end{tabular} & 2.016 & 2.019 & 2.010 & 2.014 & 2.013 & 2.011 & 2.011 \\
\hline \begin{tabular}{|l|}
$\begin{array}{l}\text { Números de } \\
\text { seguidos }\end{array}$ \\
\end{tabular} & 388 & 488 & 680 & 256 & 1.304 & 23 & 4.458 \\
\hline \begin{tabular}{|l|} 
Número de \\
tweets totales
\end{tabular} & 6.882 & 3.105 & 16.650 & 1.134 & 31.826 & 35.237 & 22.714 \\
\hline $\begin{array}{l}\text { Tweets } \\
\text { analizados }\end{array}$ & 3.200 & 3.105 & 3.200 & 1.127 & 3.184 & 3.200 & 3.199 \\
\hline $\begin{array}{l}\text { Número de } \\
\text { retweets a } \\
\text { otros } \\
\text { usuarios } \\
\end{array}$ & 1.577 & 1.781 & 1.117 & 636 & 2.061 & 175 & 418 \\
\hline $\begin{array}{l}\text { Número de } \\
\text { respuestas } \\
\text { (para ver el } \\
\text { nivel de } \\
\text { interacción) } \\
\end{array}$ & 31 & 214 & 422 & 127 & 421 & 199 & 222 \\
\hline $\begin{array}{l}\text { Media } \\
\text { tweets/día } \\
\text { (cuanto mayor } \\
\text { sea el número, } \\
\text { más activo es } \\
\text { el usuario) }\end{array}$ & 5,54 & 6,98 & 11,99 & 0,51 & 3,38 & 11,47 & 5,98 \\
\hline
\end{tabular}


RLCS, Revista Latina de Comunicación Social, 80, 89-117

[Investigación] DOI: 10.4185/RLCS-2022-1532| ISSN 1138-5820| Año 2022

\begin{tabular}{|c|c|c|c|c|c|c|c|}
\hline \multicolumn{8}{|c|}{ ACTIVIDAD DE LA CUENTA } \\
\hline & $\begin{array}{l}\text { Perfil: } \\
@ \text { SanidadGobCan }\end{array}$ & \begin{tabular}{|l|} 
Perfil: \\
@JuntaEx_Sanidad
\end{tabular} & $\begin{array}{l}\text { Perfil: } \\
\text { @saludand }\end{array}$ & $\begin{array}{l}\text { Perfil: } \\
\text { @SaludMadrid }\end{array}$ & $\begin{array}{l}\text { Perfil: } \\
\text { @astursalud }\end{array}$ & $\begin{array}{l}\text { Perfil: } \\
\text { @osakidetzaEJGV }\end{array}$ & $\begin{array}{l}\text { Perfil: } \\
\text { @SaludPublicaAra }\end{array}$ \\
\hline Análisis & $\begin{array}{l}320007 \text { Marzo } \\
2020-06 \text { Diciembre } \\
2020\end{array}$ & $\begin{array}{l}320028 \text { Marzo } 2018- \\
06 \text { Diciembre } 2020\end{array}$ & $\begin{array}{l}320021 \text { Abril } \\
2020-06 \\
\text { Diciembre } \\
2020\end{array}$ & $\begin{array}{l}274001 \\
\text { Febrero 2018- } \\
06 \text { Diciembre } \\
2020\end{array}$ & $\begin{array}{l}320025 \\
\text { Febrero } 2019 \text { - } \\
06 \text { Diciembre } \\
2020\end{array}$ & $\begin{array}{l}320002 \text { Octubre } \\
2019-06 \text { Diciembre } \\
2020\end{array}$ & $\begin{array}{l}265409 \text { Febrero } \\
2011-06 \text { Diciembre } \\
2020\end{array}$ \\
\hline Nivel & Regional & Regional & Regional & Regional & Regional & Regional & Regional \\
\hline $\begin{array}{l}\text { Idioma de } \\
\text { publicación }\end{array}$ & Español & Español & Español & Español & Español & Español y Euskera & Español \\
\hline $\begin{array}{l}\text { Año en el que } \\
\text { se unió a } \\
\text { Twitter }\end{array}$ & 2.019 & 2.013 & 2.011 & 2.018 & 2012 & 2010 & 2011 \\
\hline $\begin{array}{l}\text { Números de } \\
\text { seguidos }\end{array}$ & 82 & 73 & 1.239 & 112 & 524 & 72 & 420 \\
\hline $\begin{array}{l}\text { Número de } \\
\text { tweets totales }\end{array}$ & 4657 & 7.365 & 41.762 & 2.741 & 7.908 & 10.138 & 2656 \\
\hline $\begin{array}{l}\text { Tweets } \\
\text { analizados }\end{array}$ & 3.200 & 3.200 & 3200 & 2740 & 3200 & 3200 & 2654 \\
\hline $\begin{array}{l}\text { Número de } \\
\text { retweets a } \\
\text { otros } \\
\text { usuarios }\end{array}$ & 420 & 727 & 2455 & 559 & 1746 & 621 & 1482 \\
\hline $\begin{array}{l}\text { Número de } \\
\text { respuestas } \\
\text { (para ver el } \\
\text { nivel de } \\
\text { interacción) }\end{array}$ & 770 & 1.446 & 8 & 453 & 450 & 1165 & 121 \\
\hline $\begin{array}{l}\text { Media } \\
\text { tweets/día } \\
\text { (cuanto mayor } \\
\text { sea el número, } \\
\text { más activo es } \\
\text { el usuario) }\end{array}$ & 11,64 & 2,37 & 13,91 & 2,63 & 4,92 & 7,41 & 0,74 \\
\hline
\end{tabular}


RLCS, Revista Latina de Comunicación Social, 80, 89-117

[Investigación] DOI: 10.4185/RLCS-2022-1532| ISSN 1138-5820| Año 2022

\begin{tabular}{|c|c|c|c|c|c|c|c|}
\hline \multicolumn{8}{|c|}{ ACTIVIDAD DE LA CUENTA } \\
\hline & $\begin{array}{l}\text { Perfil: } \\
\text { @icscat }\end{array}$ & $\begin{array}{l}\text { Perfil: } \\
@ \text { @snsalud_na }\end{array}$ & \begin{tabular}{|l} 
Perfil: \\
@ibsalut
\end{tabular} & \begin{tabular}{|l} 
Perfil: \\
$@$ Murciasalud
\end{tabular} & $\begin{array}{l}\text { Perfil: } \\
\text { @riojasalud }\end{array}$ & $\begin{array}{l}\text { Perfil: } \\
\text { @Ingesa_Ceuta }\end{array}$ & $\begin{array}{l}\text { Perfil: } \\
\text { @Ingesa_Melilla }\end{array}$ \\
\hline Análisis & $\begin{array}{l}319824 \\
\text { Marzo } \\
2020-06 \\
\text { Diciembre } \\
2020\end{array}$ & $\begin{array}{l}320016 \\
\text { Septiembre } \\
2019-06 \\
\text { Diciembre } \\
2020\end{array}$ & $\begin{array}{l}320029 \\
\text { Septiembre } \\
2016-06 \\
\text { Diciembre } \\
2020\end{array}$ & $\begin{array}{l}319914 \\
\text { Diciembre } \\
2018-06 \\
\text { Diciembre } \\
2020\end{array}$ & $\begin{array}{l}319911 \text { Enero } \\
2017-06 \\
\text { Diciembre2020 }\end{array}$ & $\begin{array}{l}318126 \text { Mayo } \\
2017-06 \\
\text { Diciembre } 2020\end{array}$ & $\begin{array}{l}319911 \text { Junio } \\
2017-06 \\
\text { Diciembre } 2020\end{array}$ \\
\hline Nivel & Regional & Regional & Regional & Regional & Regional & Regional & Regional \\
\hline $\begin{array}{l}\text { Idioma de } \\
\text { publicación }\end{array}$ & $\begin{array}{c}\text { Español y } \\
\text { Catalán }\end{array}$ & Español & Español & Español & Español & Español & Español \\
\hline $\begin{array}{l}\text { Año en el que se } \\
\text { unió a Twitter }\end{array}$ & 2010 & 2010 & 2012 & 2010 & 2009 & 2011 & 2011 \\
\hline $\begin{array}{l}\text { Números de } \\
\text { seguidos }\end{array}$ & 3957 & 935 & 907 & 159 & 574 & 274 & 210 \\
\hline $\begin{array}{l}\text { Número de tweets } \\
\text { totales }\end{array}$ & 35870 & 12366 & 4489 & 11765 & 6470 & 5320 & 7440 \\
\hline Tweets analizados & 3198 & 3200 & 3200 & 3199 & 3199 & 3181 & 3199 \\
\hline $\begin{array}{l}\text { Número de retweets } \\
\text { a otros usuarios }\end{array}$ & 163 & 836 & 2562 & 1754 & 459 & 1556 & 2724 \\
\hline $\begin{array}{l}\text { Número de } \\
\text { respuestas (para } \\
\text { ver el nivel de } \\
\text { interacción) }\end{array}$ & 761 & 623 & 91 & 20 & 13 & 240 & 57 \\
\hline $\begin{array}{l}\text { Media tweets/día } \\
\text { (cuanto mayor sea el } \\
\text { número, más activo } \\
\text { es el usuario) }\end{array}$ & 12,4 & 7,14 & 2,09 & 4,42 & 2,24 & 2,46 & 5,87 \\
\hline
\end{tabular}


RLCS, Revista Latina de Comunicación Social, 80, 89-117

[Investigación] DOI: 10.4185/RLCS-2022-1532| ISSN 1138-5820| Año 2022

\begin{tabular}{|c|c|c|c|c|c|c|c|}
\hline \multicolumn{8}{|c|}{ IMPACTO DE LA CUENTA } \\
\hline & $\begin{array}{l}\text { Perfil: } \\
\text { @sanidadexunta }\end{array}$ & $\begin{array}{l}\text { Perfil: } \\
\text { @SaludCantabria }\end{array}$ & $\begin{array}{l}\text { Perfil: } \\
\text { @sanidadgob }\end{array}$ & \begin{tabular}{|l} 
Perfil: \\
@INGESAnidad
\end{tabular} & $\begin{array}{l}\text { Perfil: } \\
\text { @SanidadCLM }\end{array}$ & \begin{tabular}{|l|} 
Perfil: \\
@Salud_JCYL
\end{tabular} & $\begin{array}{l}\text { Perfil: } \\
\text { @GVAsanitat }\end{array}$ \\
\hline $\begin{array}{l}\text { Número de } \\
\text { seguidores }\end{array}$ & 8.318 & 4.165 & 600.349 & 583 & 16.292 & 28.488 & 59.054 \\
\hline $\begin{array}{l}\text { Proporción de } \\
\text { seguidores } \\
\text { por persona } \\
\text { tardía (mide el } \\
\text { nivel de } \\
\text { influencia) }\end{array}$ & 21 & 8,53 & 883 & 2,28 & 12 & 1238 & 13 \\
\hline $\begin{array}{l}\text { Número de } \\
\text { tweets } \\
\text { retuiteados } \\
\text { por otros } \\
\text { usuarios }\end{array}$ & 1.402 & 1.067 & 1.954 & 199 & 756 & 2.565 & 2.640 \\
\hline $\begin{array}{l}\text { Número de } \\
\text { veces que se } \\
\text { han retuiteado } \\
\text { los tweets }\end{array}$ & 6.333 & 10.289 & 295.909 & 488 & 13.273 & 27.090 & 76.854 \\
\hline $\begin{array}{l}\text { Media } \\
\text { retweets/ } \\
\text { tweets }\end{array}$ & $43,81 \%$ & $57,00 \%$ & $61,10 \%$ & $17,66 \%$ & 23,74 & 80,16 & $82,53 \%$ \\
\hline $\begin{array}{l}\text { Favoritos } \\
\text { recibidos de } \\
\text { otros } \\
\text { usuarios }\end{array}$ & 1.405 & 1.212 & 2.023 & 143 & 911 & 2.675 & 2.698 \\
\hline $\begin{array}{l}\text { Número de } \\
\text { veces } \\
\text { marcado } \\
\text { como favorito } \\
\text { por otros }\end{array}$ & 9.060 & 18.779 & 450.544 & 398 & 14.707 & 34.556 & 128.209 \\
\hline $\begin{array}{l}\text { media } \\
\text { favoritos/ } \\
\text { tweets }\end{array}$ & $43,91 \%$ & $39,00 \%$ & $63,2 \% \%$ & $12,69 \%$ & 28,61 & 83,59 & $84,34 \%$ \\
\hline $\begin{array}{l}\text { Eficacia } \\
\text { (retweets de } \\
\text { otros y } \\
\text { favoritos de } \\
\text { otros hacia él) } \\
\text { / Número total } \\
\text { de tweets }\end{array}$ & $87,72 \%$ & $71,22 \%$ & $124,28 \%$ & $30,35 \%$ & $52,36 \%$ & $163,75 \%$ & $166,86 \%$ \\
\hline $\begin{array}{l}\text { Hashtags } \\
\text { totales }\end{array}$ & 3.688 & 2.749 & 2.822 & 715 & 476 & 6.474 & 2.294 \\
\hline $\begin{array}{l}\text { Menciones } \\
\text { hechas al } \\
\text { perfil }\end{array}$ & 149 & 1.518 & 1.023 & 472 & 938 & 2.199 & 2.435 \\
\hline $\begin{array}{l}N^{\circ} \text { Promedio } \\
\text { de menciones } \\
\text { por tweet }\end{array}$ & 0,05 & 0,49 & 0,32 & 0,42 & 0,29 & 0,69 & 0,76 \\
\hline
\end{tabular}


RLCS, Revista Latina de Comunicación Social, 80, 89-117

[Investigación] DOI: 10.4185/RLCS-2022-1532| ISSN 1138-5820| Año 2022

\begin{tabular}{|c|c|c|c|c|c|c|c|}
\hline \multicolumn{8}{|c|}{ IMPACTO DE LA CUENTA } \\
\hline & $\begin{array}{l}\text { Perfil: } \\
@ \text { SanidadGobCan }\end{array}$ & $\begin{array}{l}\text { Perfil: } \\
@ \text { @JuntaEx_Sanidad }\end{array}$ & $\begin{array}{l}\text { Perfil: } \\
\text { @saludand }\end{array}$ & $\begin{array}{l}\text { Perfil: } \\
\text { @SaludMadrid }\end{array}$ & $\begin{array}{l}\text { Perfil: } \\
@ \text { astursalud }\end{array}$ & $\begin{array}{l}\text { Perfil: } \\
\text { @osakidetzaEJGV }\end{array}$ & $\begin{array}{l}\text { Perfil: } \\
@ \text { SaludPublicaAra }\end{array}$ \\
\hline $\begin{array}{l}\text { Número de } \\
\text { seguidores }\end{array}$ & 24.637 & 6.080 & 43.039 & 66.289 & 14.728 & 25.213 & 5.091 \\
\hline $\begin{array}{l}\text { Proporción de } \\
\text { seguidores } \\
\text { por persona } \\
\text { tardía (mide el } \\
\text { nivel de } \\
\text { influencia) }\end{array}$ & 300 & 83 & 35 & 543 & 28 & 350 & 12 \\
\hline $\begin{array}{l}\text { Número de } \\
\text { tweets } \\
\text { retuiteados } \\
\text { por otros } \\
\text { usuarios }\end{array}$ & 2323 & 1.525 & 713 & 1.918 & 1265 & 2238 & 853 \\
\hline $\begin{array}{l}\text { Número de } \\
\text { veces que se } \\
\text { han retuiteado } \\
\text { los tweets }\end{array}$ & 50255 & 4.108 & 10.349 & 105.968 & 25.767 & 28473 & 5505 \\
\hline $\begin{array}{l}\text { Media } \\
\text { retweets/ } \\
\text { tweets }\end{array}$ & $72,59 \%$ & $47,66 \%$ & $22,28 \%$ & $70,00 \%$ & $39,53 \%$ & $69,94 \%$ & $32,14 \%$ \\
\hline $\begin{array}{l}\text { Favoritos } \\
\text { recibidos de } \\
\text { otros } \\
\text { usuarios }\end{array}$ & 2594 & 1.584 & 736 & 2.004 & 1359 & 2416 & 602 \\
\hline $\begin{array}{l}\text { Número de } \\
\text { veces } \\
\text { marcado } \\
\text { como favorito } \\
\text { por otros }\end{array}$ & 115.700 & 5.103 & 17.084 & 135.417 & 51040 & 37805 & 4905 \\
\hline $\begin{array}{l}\text { media } \\
\text { favoritos/ } \\
\text { tweets }\end{array}$ & $81,06 \%$ & $49,50 \%$ & $23,00 \%$ & $73,14 \%$ & $42,47 \%$ & $75,50 \%$ & $22,68 \%$ \\
\hline $\begin{array}{l}\text { Eficacia } \\
\text { (retweets de } \\
\text { otros y } \\
\text { favoritos de } \\
\text { otros hacia él) } \\
\text { / Número total } \\
\text { de tweets }\end{array}$ & $153,66 \%$ & $97,16 \%$ & $45,28 \%$ & $143,14 \%$ & $82,00 \%$ & $145,44 \%$ & $54,82 \%$ \\
\hline $\begin{array}{l}\text { Hashtags } \\
\text { totales }\end{array}$ & 2400 & 1.394 & 825 & 5.841 & 2020 & 697 & 1431 \\
\hline $\begin{array}{l}\text { Menciones } \\
\text { hechas al } \\
\text { perfil }\end{array}$ & 1367 & 3.797 & 144 & 1.288 & 1211 & 255 & 823 \\
\hline $\begin{array}{l}N^{\circ} \text { Promedio } \\
\text { de menciones } \\
\text { por tweet }\end{array}$ & 0,43 & 1,19 & 0,05 & 0,47 & 0,38 & 0,08 & 0,31 \\
\hline
\end{tabular}


RLCS, Revista Latina de Comunicación Social, 80, 89-117

[Investigación] DOI: 10.4185/RLCS-2022-1532| ISSN 1138-5820| Año 2022

\begin{tabular}{|c|c|c|c|c|c|c|c|}
\hline \multicolumn{8}{|c|}{ IMPACTO DE LA CUENTA } \\
\hline & $\begin{array}{l}\text { Perfil: } \\
\text { @icscat }\end{array}$ & $\begin{array}{l}\text { Perfil: } \\
\text { @snsalud_na }\end{array}$ & $\begin{array}{l}\text { Perfil: } \\
\text { @ibsalut }\end{array}$ & $\begin{array}{l}\text { Perfil: } \\
\text { @Murciasalud }\end{array}$ & $\begin{array}{l}\text { Perfil: } \\
\text { @riojasalud }\end{array}$ & $\begin{array}{l}\text { Perfil: } \\
\text { @Ingesa_Ceuta }\end{array}$ & $\begin{array}{l}\text { Perfil: } \\
\text { @Ingesa_Melilla }\end{array}$ \\
\hline \begin{tabular}{|l|}
$\begin{array}{l}\text { Número de } \\
\text { seguidores }\end{array}$ \\
\end{tabular} & 56928 & 9022 & 7802 & 17579 & 5870 & 2403 & 2547 \\
\hline $\begin{array}{l}\text { Proporción de } \\
\text { seguidores por } \\
\text { persona tardía } \\
\text { (mide el nivel de } \\
\text { influencia) }\end{array}$ & 14 & 9,65 & 8,6 & 111 & 10 & 8,77 & 12 \\
\hline $\begin{array}{l}\text { Número de tweets } \\
\text { retuiteados por } \\
\text { otros usuarios }\end{array}$ & 2691 & 1553 & 593 & 1431 & 1321 & 997 & 394 \\
\hline $\begin{array}{l}\text { Número de veces } \\
\text { que se han } \\
\text { retuiteado los } \\
\text { tweets }\end{array}$ & 25402 & 8222 & 6759 & 34786 & 3308 & 4277 & 1700 \\
\hline $\begin{array}{l}\text { Media retweets/ } \\
\text { tweets }\end{array}$ & $84,15 \%$ & $48,53 \%$ & $18,53 \%$ & $44,73 \%$ & $41,29 \%$ & $31,34 \%$ & $12,32 \%$ \\
\hline $\begin{array}{l}\text { Favoritos recibidos } \\
\text { de otros usuarios }\end{array}$ & 2966 & 1644 & 605 & 1443 & 1524 & 977 & 373 \\
\hline $\begin{array}{l}\text { Número de veces } \\
\text { marcado como } \\
\text { favorito por otros }\end{array}$ & 72520 & 11191 & 9393 & 45540 & 4620 & 4536 & 1623 \\
\hline $\begin{array}{l}\text { media favoritos/ } \\
\text { tweets }\end{array}$ & $92,75 \%$ & $51,38 \%$ & $18,91 \%$ & $45,11 \%$ & $47,64 \%$ & $30,71 \%$ & $11,66 \%$ \\
\hline $\begin{array}{l}\text { Eficacia (retweets } \\
\text { de otros y favoritos } \\
\text { de otros hacia él) / } \\
\text { Número total de } \\
\text { tweets }\end{array}$ & $176,89 \%$ & $99,91 \%$ & $37,44 \%$ & $89,84 \%$ & $88,93 \%$ & $62,06 \%$ & $23,98 \%$ \\
\hline Hashtags totales & 5828 & 4969 & 320 & 4632 & 2981 & 230 & 300 \\
\hline $\begin{array}{l}\text { Menciones hechas } \\
\text { al perfil }\end{array}$ & 4454 & 1170 & 869 & 617 & 542 & 207 & 50 \\
\hline $\begin{array}{l}N^{\circ} \text { Promedio de } \\
\text { menciones por } \\
\text { tweet }\end{array}$ & 1,39 & 0,37 & 0,27 & 0,19 & 0,17 & 0,07 & 0,02 \\
\hline
\end{tabular}

Fuente: elaboración propia 
RLCS, Revista Latina de Comunicación Social, 80, 89-117

[Investigación] DOI: 10.4185/RLCS-2022-1532| ISSN 1138-5820| Año 2022

\section{ANEXO II}

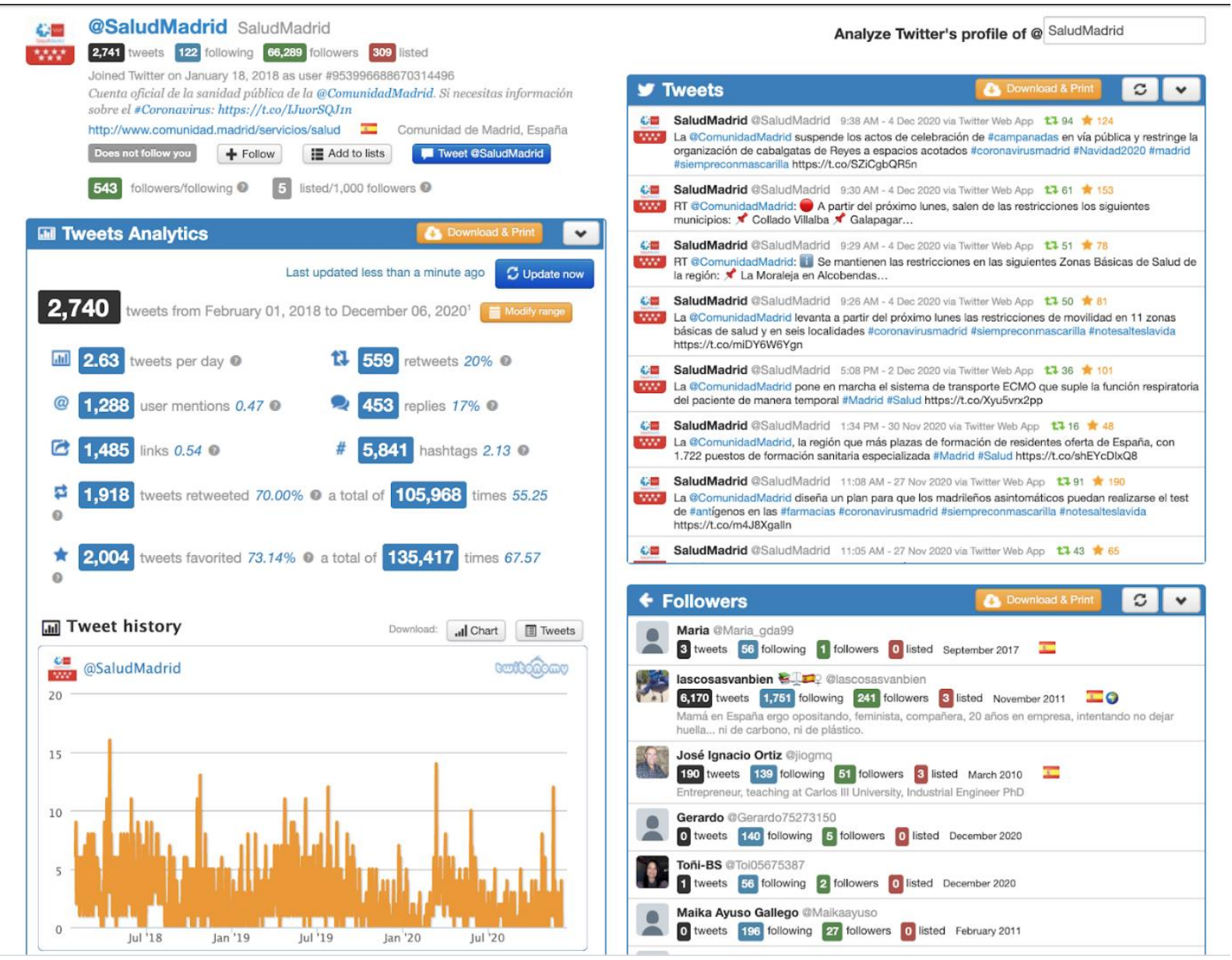

Figura 3. Imagen de la información extraída de uno de los perfiles a través de Twitonomy Fuente: elaboración propia a través de Twitonomy 